SEPTEMBER 1999, PAGES 401-432

\title{
A STUDY OF THE EFFECTIVE PROPERTIES OF MASS AND STIFFNESS MICROSTRUCTURES- A MULTIRESOLUTION APPROACH
}

\author{
BY \\ BEN ZION STEINBERG (Department of Interdisciplinary Studies, Faculty of Engineering, \\ Tel-Aviv University, Israel) \\ AND
}

JOHN J. McCOY (School of Engineering, The Catholic University of America, Washington, DC)

\begin{abstract}
The theory of multiresolution decomposition and wavelets is used to study the effective properties of a thin elastic plate with surface mass density or stiffness heterogeneity, subjected to time-harmonic forcing. The heterogeneity possesses micro- and macro-scale variations, and has a macroscale outer dimension. It is shown that the microscale mass variation has practically no effect on the macroscale plate response, whereas microscale stiffness variation can have a significant effect. We derive an effective constitutive relation pertaining to a microscale stiffness variation. It is shown that it is possible to synthesize classes of different stiffness microstructures that have the same footprint on the macroscale component of the plate response. An effective, smooth, stiffness heterogeneity associated with the classes is developed. The results are first derived analytically and then supported by numerical simulations.
\end{abstract}

1. Introduction. A significant and fundamental problem at the intersection of mechanics and materials science is the accommodation of a heterogeneity that exists over a broad range of length scales.

Two aspects of the problem are significant. The first is the task of describing the heterogeneity itself. Improvements in instrumentation and automatic data collection methods allow for a point-by-point description of a material substructure across a section of a specimen, at resolutions that were unthinkable a short time ago. Faced with vast amounts of data, the researcher requires signal processing algorithms for extracting that information, which enables improved predictive capability of the behavior of material specimens, and the design of materials microstructures. The decomposition of the total variation in the point-by-point description, according to the length-scales on which the variation can be observed is an effective strategy for the extraction of information.

Received August 18, 1995.

1991 Mathematics Subject Classification. Primary 34-XX, 35-XX, 45-XX, 73-XX, 42-XX.

(C)1999 Brown University 
The second aspect of the problem of multiple length-scales is the task of separating processes according to the scales on which they operate. A complexity in estimating the response of material systems resides in the presence of multiple processes, all operating simultaneously and on different length-scales. Due to an inherent nonlinearity in the dependence of the response of material systems on measures of material heterogeneity, as well as any nonlinearity in the individual operative processes, there is a highly complicated across-length-scale coupling. Tools for addressing this across-length-scale coupling are an important missing link for the systematic application of mechanics to materials science.

The theory of a multiresolution decomposition using discrete orthogonal wavelets [3][4] provides a new framework for describing variability which can be observed on a broad range of length scales. Developed primarily by mathematicians and electrical engineers interested in signal processing, signal compression, and signal communication [5], it is this application of wavelets that translates most obviously for use in materials science. The framework of a multiresolution decomposition can also be applied for separating processes according to length-scales. While a less obvious application, it is this one that is potentially the more far-reaching in the context of mechanics and materials science. It is this application that motivates the reported study.

The specific experiment addressed applies to the dynamical response of a thin, linearly elastic plate containing a local region of material property variation. Multiple scales defined by the material variation consist of a macroscale, defined by the extent of the region containing the heterogeneity, and a microscale, defined as a small fraction of the macroscale (say $\frac{1}{30}$ to $\frac{1}{50}$ ). The continuous material property variation is accepted as observable on both the macroscale and the microscale. A discussion of an experiment's length scales is incomplete without consideration of those of the problem forcing. It is convenient to discuss the problem forcing in terms of the plate response that would be obtained in the absence of the local region of heterogeneity; i.e., in terms of a homogeneous background plate. We accept a plate forcing such that the homogenous background plate response, in the vicinity of the local region of heterogeneity, is observable only on the macroscale.

Intuitively, the local region of heterogeneity introduces two changes in the homogeneous background plate response. One change refers to modifications of the macroscale response variation of the homogeneous background plate, which can also be observed on the macroscale, and the second is the addition of a response variation that can be observed on the microscale. Because the interaction of the plate response and the material heterogeneity is inherently nonlinear, even for a theory of plates that is classified as linear, there exists an across-scale-coupling by which the modifications to the macroscale response of the plate depend on both the macroscale and the microscale heterogeneity. What might be termed the "composite materials" problem refers to relating changes to be observed in the macroscale response of the plate, occasioned by the presence of the local region of multiscale heterogeneity. This is the motivating problem of the reported study.

The literature of the micromechanics of composite materials, to which the present paper is a contribution, is extensive. To set a context, in a more commonly addressed 
problem the region of heterogeneity extends over the entire domain of the plate and the continuous material property variation is accepted as observable only on a microscale. The "specimen" macroscale, of the above articulation, is accepted as unboundedly large. A finite "experiment" macroscale is provided by the problem forcing. As suggested above, the problem forcing is conveniently described by what would be the plate response in the absence of the variation in the material property field. Further, for the more commonly addressed micromechanics of composite materials problem, this background plate response is described as a propagating plane wave. On the condition that the wavelength of this background plane wave is large compared to the material microscale, one can show that the macroscale component of the plate response in the presence of the material microscale, is also described by a propagating plane wave. The prediction problem is to relate the parameters of this modified plane wave to a description of the material microscale. The modified macroscale component of the plate response can be associated with the response of an "effective" homogeneous plate.

In contrasting the more commonly addressed "composite materials" problem with the generalization of the present study, the most significant feature would be the coupling effects of material heterogeneity observable on the macroscale and on the microscale. The elimination of macroscale material variation in the more commonly addressed problem precludes any discussion of this coupling. It is this feature, then, that is the main contribution of the reported study.

Allowing both a macroscale and a microscale specimen heterogeneity requires a mathematical framework that allows their separation. The "models" of material variation that are the bases for previous studies - that of a statistically homogeneous stochastic process and that of a periodic cell-cannot be this framework. The theory of a multiresolution decomposition in terms of orthogonal wavelets can. A new formulation of the problem associated with a multiple-scale heterogeneity, which is a priori tuned to govern the macroscale response component, is developed. This formulation holds for an arbitrary continuum of scales. At this level, two fundamental questions can be posed as regards the footprint of the heterogeneity in the macroscale response. The first question is: What types of heterogeneity have a footprint? For those heterogeneities for which a footprint exists, one might expect that only limited information of the heterogeneity is required to estimate its footprint in the macroscale response. The second question is: What is this information? General asymptotic study of the new formulation, carried out for the case of a heterogeneity possessing a large gap of scales between the macro and the micro, shows how the articulated fundamental questions can be addressed. This demonstration of the potential of the new approach, and the details of the demonstration, are the main conclusions of the study. As regards the first question, we show that the degree to which a microscale heterogeneity affects the macroscale response depends on the singularity of the kernel associated with the integral equation formulation of the problem. This result is then used to address the second question.

We note that multiresolution analysis and wavelets have been used recently in [6] for numerical homogenization, i.e. for generating an equation with slowly-varying coefficients whose solution has the same large-scale behaviour as that of the original equation. Relations of this type have been pointed out and detailed also in $[1,2]$. The work in $[6]$ is 
devoted mainly to a sophisticated "decimation" process in which an efficient numerical algorithm for estimating the large-scale response component is developed. It does not address directly any of the questions articulated above.

The outline of the paper is as follows: In the next section we provide a brief description of the theory of multiresolution decomposition and the phase-space substructuring methodology. This section should make the paper self-contained for readers with some familiarity with the theory of multiresolution decomposition and orthogonal wavelets. In Sec. 3 we develop the formulation that governs the large-scale, or smoothed, plate response. We refer to this formulation as the "formulation smooth", preferring to use "smooth formulation" to imply the formulation that determines the complete plate response, made smooth by removing the small-scale variation. In Sec. 4 we discuss more precisely the concept of length scales in characterizing complex dynamical systems, making more precise the intuitive language used in describing the class of experiments of interest. Section 5 contains an implementation of the new formulation to the compositematerials mechanics problem of a thin, linearly elastic plate possessing mass or stiffness heterogeneity. Demonstrated is that a microscale variation of mass has no footprint in the large-scale response, whereas a microscale variation of stiffness can significantly affect the large-scale response. These results are first derived analytically and then demonstrated by numerical simulations. In Sec. 6 we derive an expression that determines the class of small-scale stiffness variations with identical footprints in the large-scale plate response. The results are demonstrated numerically by generating small-scale stiffness variations that are easily seen to be different, and showing that these lead to the same large-scale plate response. Concluding remarks are presented in Sec. 7.

2. Multiresolution decomposition and smoothing. The concept of multiresolution decomposition, or approximation, and its connection to the wavelet expansion have been introduced by Mallat [3]. We shall briefly summarize the essentials of this theory. Our purpose is not to provide a mathematically rigorous description of the subject, but rather to present the concept of multiresolution decomposition and wavelet expansion using intuitive notions tailored to the physics of the class of problems discussed in the present work. A detailed and rigorous mathematical treatment can be found elsewhere $[3,4]$. A physical interpretation of the concept of a multiresolution decomposition is presented first, in Sec. 2.1. A more technical representation of multiresolution decomposition suitable as a basis of the ensuing theory of effective properties is next presented in Sec. 2.2. Readers familiar with the physical interpretation of multiresolution theory can skip Sec. 2.1 since it does not present results needed for subsequent derivations. Readers familiar with the "working mathematics" of multiresolution decomposition can also skip Sec. 2.2. The rest of the paper, however, makes use of the concepts and results presented in that section.

2.1. Heuristic description. The relevance of theoretical/analytic or computational prediction models to laboratory experiments requires that the predicted physical quantitiesacoustic pressure, velocity field, energy flux, etc.-can be measured. It must be recognized, however, that such measurements cannot be interpreted as representing the corresponding quantities "at a point". The physical dimensions of sensing apparati are 


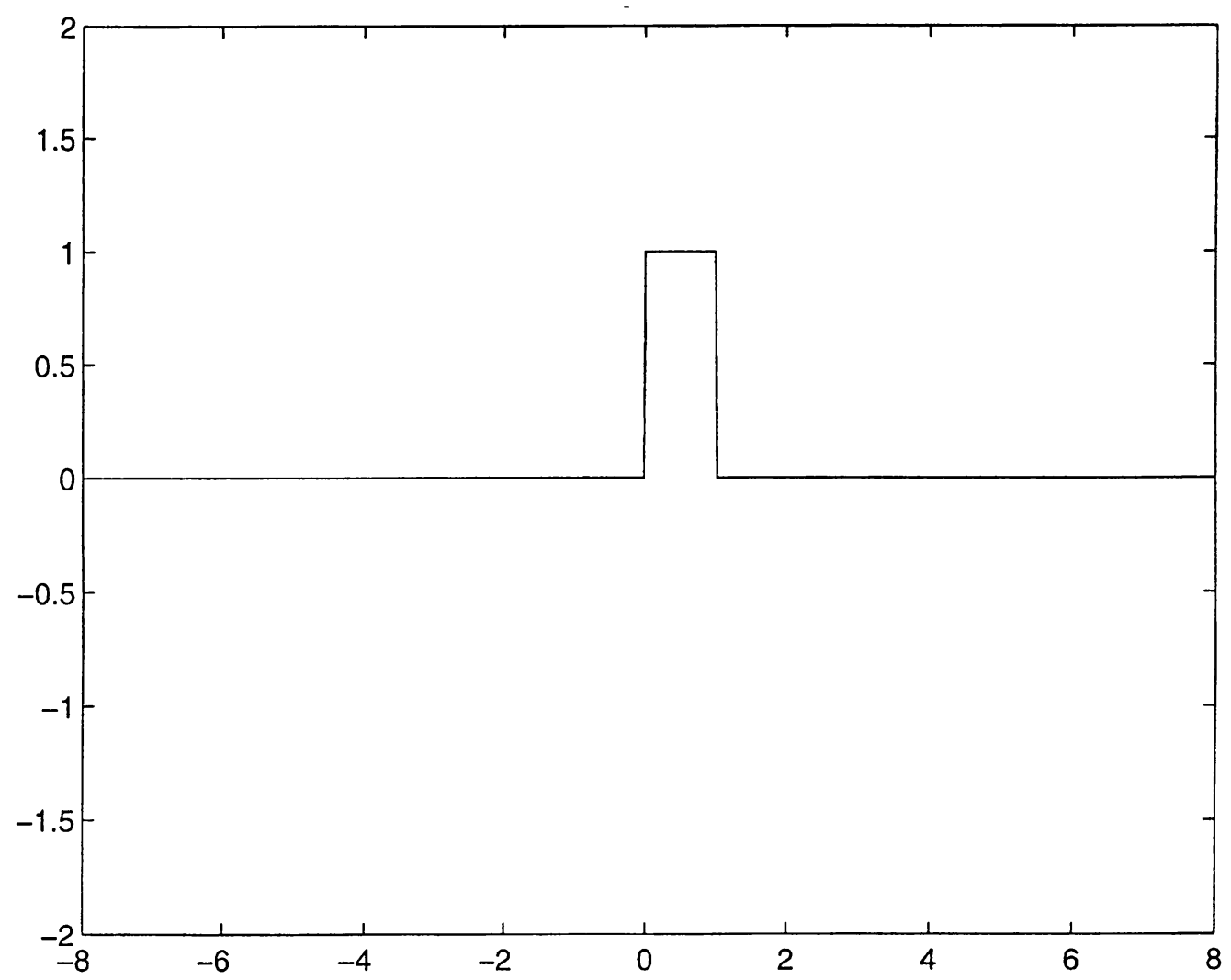

FIG. 1(a). The Haar system. The scaling function $\phi(x)$

inherently finite, and any measurement must be interpreted as a spatial average of the measured field over the neighborhood occupied by the apparatus for achieving it. The smaller the neighborhood, the closure the measurement to the ideal one taken "at a point". This can be described mathematically as multiplying the field $f$ by the "sensitivity function" $\phi$ of the measuring device, normalized by $\int \phi d x=1$, and integrating over the spatial domain. The simplest form of a realistic sensitivity function is shown in Fig. 1(a). The term "resolution" is introduced to quantify the difference between the ideal pointwise representation of the field and that provided by the measuring device schematized by $\phi$ in Fig. 1(a). Anticipating the use of wavelets, we define the resolution as the negative of a base-two logarithm of the spatial extent of $\phi$. An arbitrarily chosen standard measuring apparatus is chosen to have a resolution 0 . A measuring apparatus twice as narrow ( $\phi=2$ for $0 \leq x \leq 1 / 2$ and 0 elsewhere) has a resolution 1 , and so on.

When a field has been measured by an array of sensors with the resolution $j$, variations that are confined in space to neighborhoods smaller than $2^{-j}$ are lost. Roughly speaking, the fields that can be reconstructed from measurements taken by such arrays are constant along the intervals $n 2^{-j},(n+1) 2^{-j}$. For brevity, let us refer to the collection of all such possible reconstructions as $V_{j}$. It is clear that a reconstruction in $V_{j+1}$ requires more 
information, more measurements, and more storage space than a reconstruction in $V_{j}$. Consider, for example, an array with the resolution 1. The field reconstructions at the two adjacent detector locations $n, n+1$ in that array, say $\bar{f}_{n}$ and $\bar{f}_{n+1}$, can be presented as a single average $\frac{1}{2}\left(\bar{f}_{n}+\bar{f}_{n+1}\right)$, obtained using a detector with resolution 0 , and a correction term given by the distribution $\psi$ of Fig. 2(a) multiplied by the factor $\frac{1}{2}\left(\bar{f}_{n}-\bar{f}_{n+1}\right)$. This factor is nothing but the local difference in information needed to improve from the data at resolution 0 to the data at resolution 1. Furthermore, just as a measurement of $f$ at resolution 0 can be presented by $\int f \phi d x$, the articulated difference in information can be presented by $\int f \psi d x$, that is, a measurement taken by an apparatus with the sensitivity function $\psi$. Such a detector, in fact, measures a rough estimate of the spatial derivative of $f$. As implied by the factor $\frac{1}{2}\left(\bar{f}_{n}-\bar{f}_{N+1}\right)$, its measurements would be significant only in regions where the measured field varies rapidly on the length-scale associated with the resolution 0 . It is easily verified that $\psi$ and $\phi$ are orthogonal, i.e., $\int \phi \psi d x=0$. Thus the information measured by the corresponding detectors is disjoint. Let us refer to the collection of all such possible differences in information-between $V_{0}$ and $V_{1}$-as $O_{0}$. The difference in information between $V_{j}$ and $V_{j+1}$ is obtained in a similar way, with a properly scaled $\psi$, and the collection of all possible reconstructions of differences in information is denoted $O_{j}$. A reconstruction from $V_{j+1}$ can thus be presented as a reconstruction from $V_{j}$ plus an orthogonal term from $O_{j}$. This process can now be repeated to any degree of accuracy (or resolution). A reconstruction at resolution $k$ can be obtained either by directly applying measurements at that resolution, or alternatively, by using a reconstruction at a reference resolution $j$ plus a series of mutually orthogonal differences in information, sequentially improving from coarse $(j)$ to fine $(k)$.

The theory of multiresolution decomposition is the mathematical framework within which this physical description can be made precise. It also provides more favorable alternatives to $\phi$ (termed "scaling function") and $\psi$ (termed "wavelet") by generalizing the spaces $V_{j}$ and $O_{j}$.

2.2. Multiresolution decomposition. Let $\phi(x)$ and $\psi(x)$ be the scaling function and wavelet associated with an orthogonal multiresolution decomposition (MRD) of $L_{2}(R)$ $[3,4]$. An approximation of a function $f(x)$ at a resolution $k$ can be written as the sum of two mutually orthogonal functions, namely a smooth $\left(f^{s}\right)$ component and a detail $\left(f^{d}\right)$ component. We have

$$
f(x)=f^{s}(x)+f^{d}(x)
$$

where

$$
\begin{aligned}
& f^{s}(x)=\mathbf{P}_{j} f(x)=\sum_{n} F_{n}^{s} \phi_{j n}(x), \quad F_{n}^{s}=\left\langle f, \phi_{j n}\right\rangle, \\
& f^{d}(x)=\mathbf{D}_{j}^{k} f(x)=\sum_{m=j}^{k-1} \sum_{n} F_{m n}^{d} \psi_{m n}(x), \quad F_{m n}^{d}=\left\langle f, \psi_{m n}\right\rangle,
\end{aligned}
$$

in which $\phi_{j n}(x)=2^{j / 2} \phi\left(2^{j} x-n\right)$ and $\psi_{m n}(x)=2^{m / 2} \psi\left(2^{m} x-n\right)$. Here, $\langle f, g\rangle$ denotes the inner product of $L_{2}(R)$, and $j<k$ is some reference resolution-a judicious choice of which depends on the physics of the problem. The scaling functions and wavelets satisfy the orthonormality relations $\left\langle\phi_{j n}, \phi_{j n^{\prime}}\right\rangle=\delta_{n n^{\prime}},\left\langle\psi_{m n}, \psi_{m^{\prime} n^{\prime}}\right\rangle=\delta_{m m^{\prime}} \delta_{n n^{\prime}}$, and 


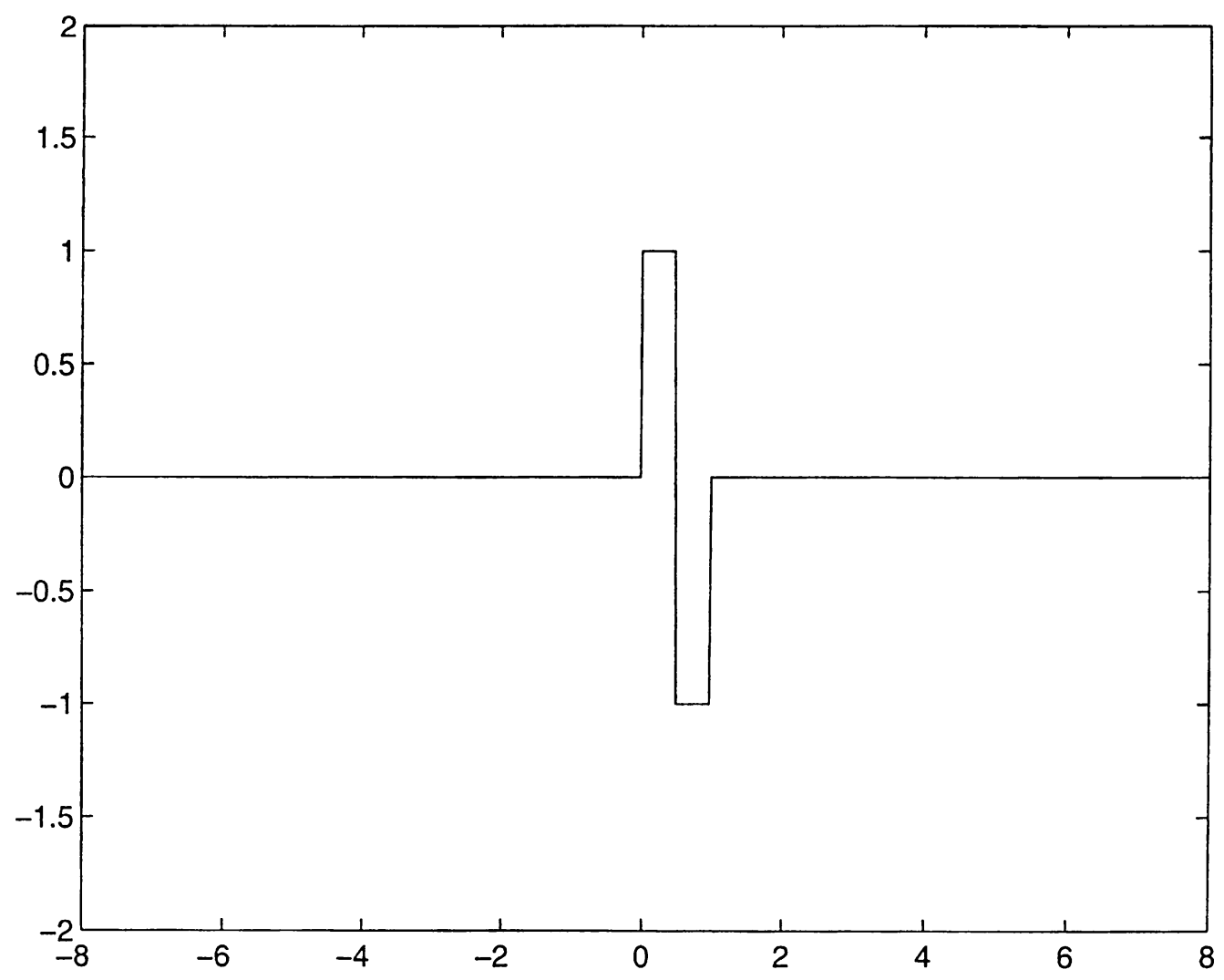

FIG. 1(b). The Haar system. The wavelet $\psi(x)$

$\left\langle\phi_{j n^{\prime}}, \psi_{m n}\right\rangle=0 \forall j \leq m$. Thus, $\mathbf{P}_{j}$ and $\mathbf{D}_{j}^{k}$ in $(2.1 \mathrm{a})-(2.1 \mathrm{~b})$ are projection operators satisfying

$$
\mathbf{D}_{j}^{k} \mathbf{P}_{j^{\prime}}=\mathbf{P}_{j^{\prime}} \mathbf{D}_{j}^{k}=0, \quad \forall j \geq j^{\prime}
$$

The scaling functions and the wavelets are spatially confined functions (i.e., $\phi$ and $\psi$ are either of compact support, or fast decreasing), centered more or less about the origin. They can be interpreted, respectively, as defining a local low-pass filter and a local bandpass filter. Examples are shown in Figs. 1 and 2. Let us define the effective supports of $\psi$ and $\phi$ as the intervals outside of which their respective values are practically zero, and denote them by $\Lambda_{\psi}$ and $\Lambda_{\phi}$. From the dilation translation relations articulated above, it follows that the terms $\psi_{m n}$ in $(2.1 \mathrm{~b})$ are situated, respectively, around the points

$$
x_{m n}=n 2^{-m},
$$

each having an effective support $2^{-m} \Lambda_{\psi}$. A similar description applies to the terms $\phi_{j n}$. The set of points in (2.3) is called the wavelet grid. The functions $f^{s}(x)$ and $f^{d}(x)$ in (2.1) can be interpreted as a locally smoothed, or averaged, description of $f(x)$ on the length-scale $2^{-j}$, and a signal describing the finer details covering length-scales ranging from $2^{-(j+1)}$ to $2^{-k}$, respectively. Here and henceforth we refer to the number $2^{-m}$ 
and the index $m$ as a length-scale and the resolution associated with it, respectively. A wavelet has $M$ vanishing moments,

$$
\int x^{m} \psi(x) d x=0, \quad m=0,1, \ldots, M-1 .
$$

For the Haar wavelet $M=1$; for the cubic-spline Battle-Lemarie wavelet $M=4[3,4]$. This parameter can be related to the regularity of the multiresolution system and to the support of the associated wavelets and scaling functions (see [5] for details and examples).

When the function $f(x)$ in the neighborhood of $x=x_{m n}$ varies slowly compared to the length-scale $2^{-m}$ and possesses $M$ first derivatives (see (2.4) for $M$ ), it is possible to derive approximate expressions for the inner products $\left\langle f, \phi_{m n}\right\rangle$ and $\left\langle f, \psi_{m n}\right\rangle$. More specifically, it can be shown that for the cubic spline Battle-Lemarie system shown in Fig. 2 (see [1]),

$$
\begin{gathered}
\left\langle f, \phi_{m n}\right\rangle=2^{-m / 2} f\left(x_{m n}\right)-\alpha 2^{-m(2 M+1 / 2)} O\left(f_{2 M}\right), \\
\left\langle f, \psi_{m n}\right\rangle=\beta 2^{-m(M+1 / 2)} f_{M I}\left(x_{m n}+2^{-m-1}\right)+\beta 2^{-m(M+5 / 2)} O\left(f_{M+2}\right),
\end{gathered}
$$

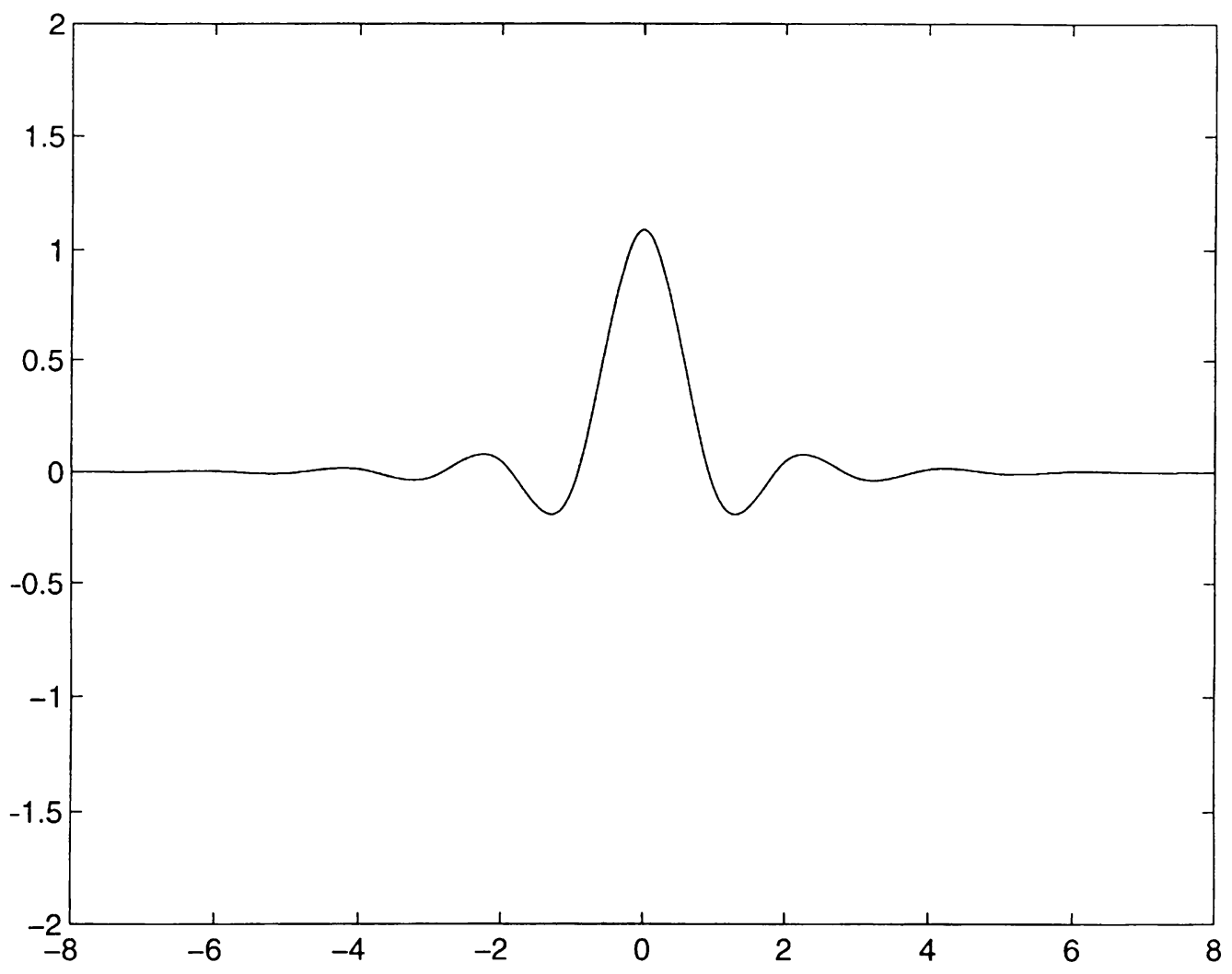

FIG. 2(a). The cubic spline Battle-Lemarie multiresolution system.

The scaling function $\phi(x)$ 


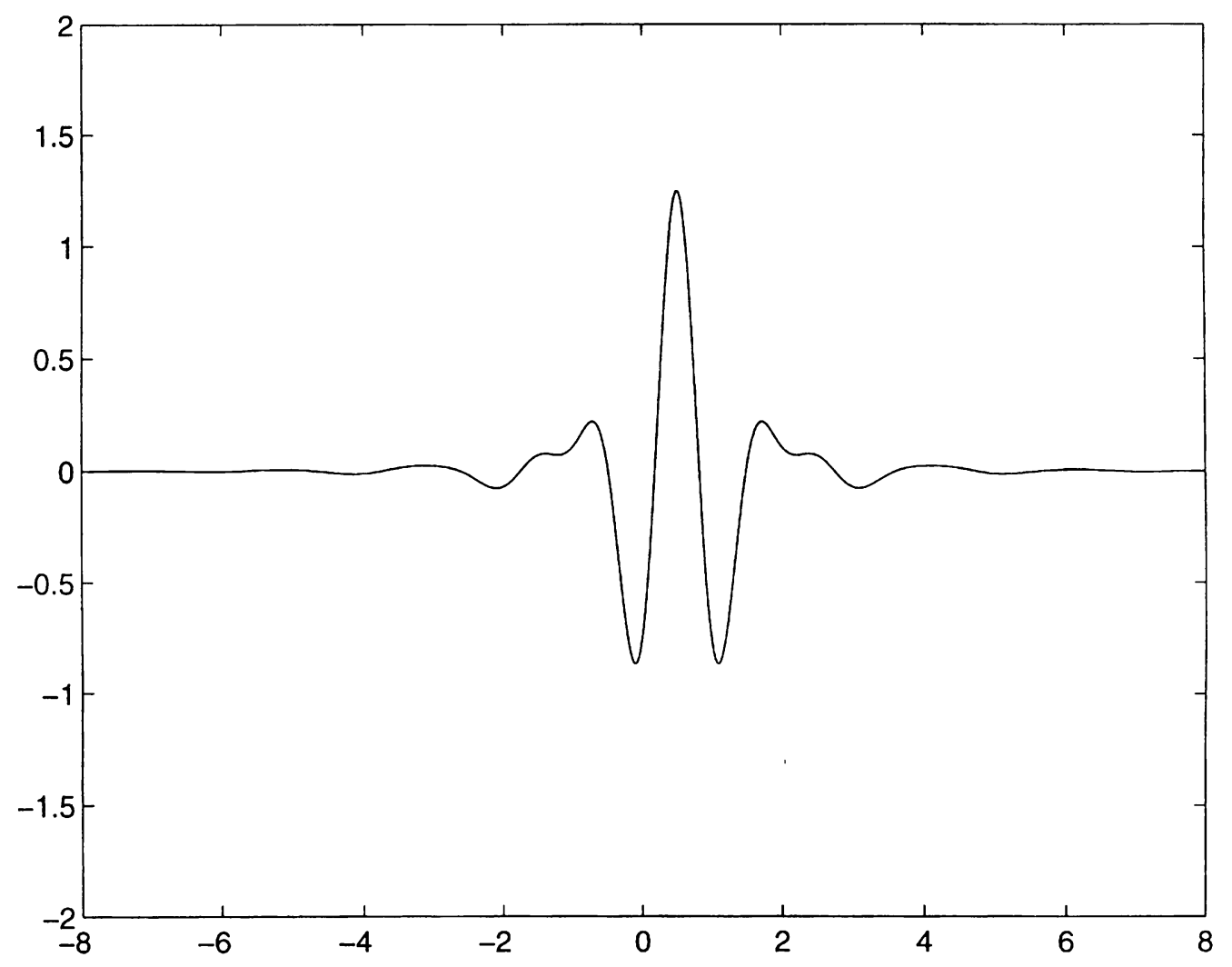

FIG. 2(b). The cubic spline Battle-Lemarie multiresolution system.

The wavelet $\psi(x)$

where $f_{M I}(x)$ is defined as

$$
f_{M}(x)=\left(i \frac{d}{d x}\right)^{M} f(x)
$$

$\alpha$ and $\beta$ are constants given by

$$
\alpha=\frac{B_{M}}{2(2 M) !} \text { and } \beta=2^{-M I}\left[\frac{\left(2^{2 M}-1\right) B_{M}}{(2 M) !}\right]^{1 / 2} .
$$

$B_{M}$ is the $M$ th Bernoulli number, and $x_{m n}$ is a point in the wavelet grid defined by Eq. (2.3). Note that for the cubic spline Battle-Lemarie system one has $M=4, B_{M}=1 / 30$, and so $\alpha, \beta \ll 1$. Thus, the remaining terms in the right-hand side of $(2.5 \mathrm{a})-(2.5 \mathrm{~b})$ are small. Furthermore, if $f_{M}=O(1)$, we get

$$
\left|\left\langle f, \psi_{m n}\right\rangle\right| \ll 1, \quad \text { for } m \gg 1,
$$

which actually states that the inner product with the wavelet is vanishingly small if the function $f$ is smooth. Similar results can derived for other multiresolution systems. 
3. The formulation smooth. The procedures that will be described here can be applied to a general linear operator equation. However, to allow for direct implementations we shall refer throughout to the Fredholm integral equation of the second kind

$$
u(x)=u_{0}(x)+\mathbf{L} u, \quad a \leq x \leq b
$$

where $\mathbf{L}$ is an integral operator defined as

$$
\mathbf{L} u=\int_{a}^{b} G\left(x-x^{\prime}\right) h\left(x^{\prime}\right) u\left(x^{\prime}\right) d x^{\prime}
$$

and where $u_{0}(x)$ is a forcing term, $u(x)$ is the response to be determined, and $G(x)$ is obtained from the Green functions of the appropriately defined background problem, usually that modeled by linear differential equations with constant coefficients. Further, $h(x)$ represents a material heterogeneity with variations occurring on both the microscale and macroscale. In the absence of the heterogeneity $h(x)$, Eq. (3.1) takes the form $u(x)=u_{0}(x)$; thus the integral equation forcing term $u_{0}(x)$ is the background system response. The operation $\mathbf{L} u$ can be represented as the background operator $\mathbf{L}_{b}$ associated with $G(x)$, operating in the function $h u$,

$$
\mathbf{L} u=\mathbf{L}_{b}(h u)
$$

where

$$
\mathbf{L}_{b} f=\int G\left(x-x^{\prime}\right) f\left(x^{\prime}\right) d x^{\prime}
$$

and it is assumed that the integration limits in (3.1a) are effected by $h$. Finally, it is usually assumed that the variations of $u_{0}(x)$ are restricted to the macroscale only.

With the MRD theory, the integral equation formulation (3.1) can be decomposed into a pair of coupled formulations governing a length-scale resolved response. We express the response field $u(x)$ as a sum of two components, namely the response smooth $u^{s}(x)$ (macroresponse) and the response detail $u^{d}(x)$ (microresponse). If $2^{-k}$ is a lower bound on the length-scales pertaining to the problem, then $u(x) \simeq \mathbf{P}_{k} u(x)$, and one can invoke $(2.1)$

$$
u(x)=u^{s}(x)+u^{d}(x)
$$

where

$$
\begin{aligned}
& u^{s}(x)=\mathbf{P}_{j} u(x)=\sum_{n=n_{1}(j)}^{n_{2}(j)} s_{n} \phi_{j n}(x), \\
& u^{d}(x)=\mathbf{D}_{j}^{k} u(x)=\sum_{m=j}^{k-1} \sum_{n=n_{1}(m)}^{n_{2}(m)} d_{m n} \psi_{m n}(x) .
\end{aligned}
$$

The former and the latter represent, respectively, the response field smoothed on a reference length-scale $2^{-j}$, and the remaining fine details. $s_{n}$ and $d_{m n}$ are yet to be determined coefficients, representing the smooth and detail parts of the response. By Eq. (2.2) $u^{s}$ and $u^{d}$ are mutually orthogonal, and by Eq. (2.4) the spatial average of $u^{d}$ vanishes. 
Substituting (3.3) into (3.1) and further operating on the resulting equation with $\mathbf{P}_{j}$ and $\mathbf{D}_{j}^{k}$, we obtain

$$
\begin{aligned}
& u^{s}(x)=u_{0}^{s}(x)+\mathbf{P}_{j} \mathbf{L} \mathbf{P}_{j} u+\mathbf{P}_{j} \mathbf{L} \mathbf{D}_{j}^{k} u, \\
& u^{d}(x)=u_{0}^{d}(x)+\mathbf{D}_{j}^{k} \mathbf{L} \mathbf{P}_{j} u+\mathbf{D}_{j}^{k} \mathbf{L} \mathbf{D}_{j}^{k} u
\end{aligned}
$$

where $u_{0}^{s}(x)$ and $u_{0}^{d}(x)$ are the mutually orthogonal smooth and detail components of the integral equation forcing term, derived from $u_{0}(x)$ via a relation similar to (3.3a) and $(3.3 \mathrm{~b})$,

$$
\begin{aligned}
& u_{0}^{s}(x)=\mathbf{P}_{j} u_{0}(x)=\sum_{n=n_{1}(j)}^{n_{2}(j)} e_{n} \phi_{j n}(x), \quad e_{n}=\left\langle\iota_{0}, \phi_{j n}\right\rangle \\
& u_{0}^{d}(x)=\mathbf{D}_{j}^{k} u_{0}(x)=\sum_{m=j}^{k-1} \sum_{n=n_{1}(m)}^{n_{2}(m)} E_{m n} \psi_{m n}(x), \quad E_{m n}=\left\langle u_{0}, \psi_{m n}\right\rangle .
\end{aligned}
$$

Since the expansion elements are orthogonal, Eqs. (3.4a) and (3.4b) can be cast into a matrix form, written for the sets of unknown coefficients $\left\{s_{n}\right\}$ and $\left\{d_{m n}\right\}$. Toward this end, we define the column vector $\vec{s}$ as the $N(j)=\left(n_{2}(j)-n_{1}(j)+1\right)$-tuple,

$$
\vec{s}=\left\{s_{n_{1}(j)}, \ldots, s_{n}, s_{n+1}, \ldots, s_{n_{2}(j)}\right\}^{\mathrm{T}}
$$

and the detail response column vector $\vec{d}$ as the stack of the scale subvectors $\vec{d}_{m}$,

$$
\vec{d}=\left\{\vec{d}_{j}, \vec{d}_{j+1}, \ldots, \vec{d}_{k-1}\right\}^{\mathrm{T}}
$$

where $\vec{d}_{m}$ is arranged in a manner similar to (3.6),

$$
\vec{d}_{m}=d_{m n_{1}(m)}, \ldots, d_{m n_{2}(m)} .
$$

Equations (3.4a) and (3.4b) can now be expressed as

$$
\left(\begin{array}{cc}
\mathbf{I}-\mathbf{\Phi} & -\mathbf{C} \\
-\overline{\mathbf{C}} & \mathbf{I}-\boldsymbol{\Psi}
\end{array}\right)\left(\begin{array}{l}
\vec{s} \\
\vec{d}
\end{array}\right)=\left(\begin{array}{c}
\vec{e} \\
\vec{E}
\end{array}\right)
$$

where $\mathbf{I}$ is an identity matrix, $\vec{e}$ and $\vec{E}$ are the excitation smooth and detail column vectors, whose elements are the respective coefficients $e_{n}$ and $E_{m n}$ in Eqs. (3.5a) and (3.5b), ordered similarly with $\vec{s}$ and $\vec{d}$ in (3.6) and (3.7a)-(3.7b). $\Phi, \Psi, \mathbf{C}$, and $\overline{\mathbf{C}}$ are matrix operators whose elements are given, respectively, by

$$
\begin{aligned}
\Phi_{n^{\prime}, n} & =\left\langle\mathbf{P}_{j} \mathbf{L} \mathbf{P}_{j} \phi_{j n}, \phi_{j n^{\prime}}\right\rangle=\left\langle\mathbf{L} \phi_{j n}, \phi_{j n^{\prime}}\right\rangle, \\
\Psi_{m^{\prime} n^{\prime}, m n} & =\left\langle\mathbf{D}_{j}^{k} \mathbf{L} \mathbf{D}_{j}^{k} \psi_{m n}, \psi_{m^{\prime} n^{\prime}}\right\rangle=\left\langle\mathbf{L} \psi_{m n}, \psi_{m^{\prime} n^{\prime}}\right\rangle, \\
C_{n^{\prime}, m n} & =\left\langle\mathbf{P}_{j} \mathbf{L D}_{j}^{k} \psi_{m n}, \phi_{j n^{\prime}}\right\rangle=\left\langle\mathbf{L} \psi_{m n}, \phi_{j n^{\prime}}\right\rangle, \\
\bar{C}_{m^{\prime} n^{\prime}, n} & =\left\langle\mathbf{D}_{j}^{k} \mathbf{L} \mathbf{P}_{j} \phi_{j n}, \psi_{m^{\prime} n^{\prime}}\right)=\left\langle\mathbf{L} \phi_{j n}, \psi_{m^{\prime} n^{\prime}}\right\rangle .
\end{aligned}
$$

The elements are ordered in their respective matrices in a manner consistent with Eqs. (3.6)-(3.7b) - primed indices counting the rows, and location indices $(n)$ running fasteras schematized in Fig. 3.

Equation (3.8) provides the starting point for a self-consistent multiresolution study of scattering problems (see [1, 2] and [7]) and for a self-consistent development of the 


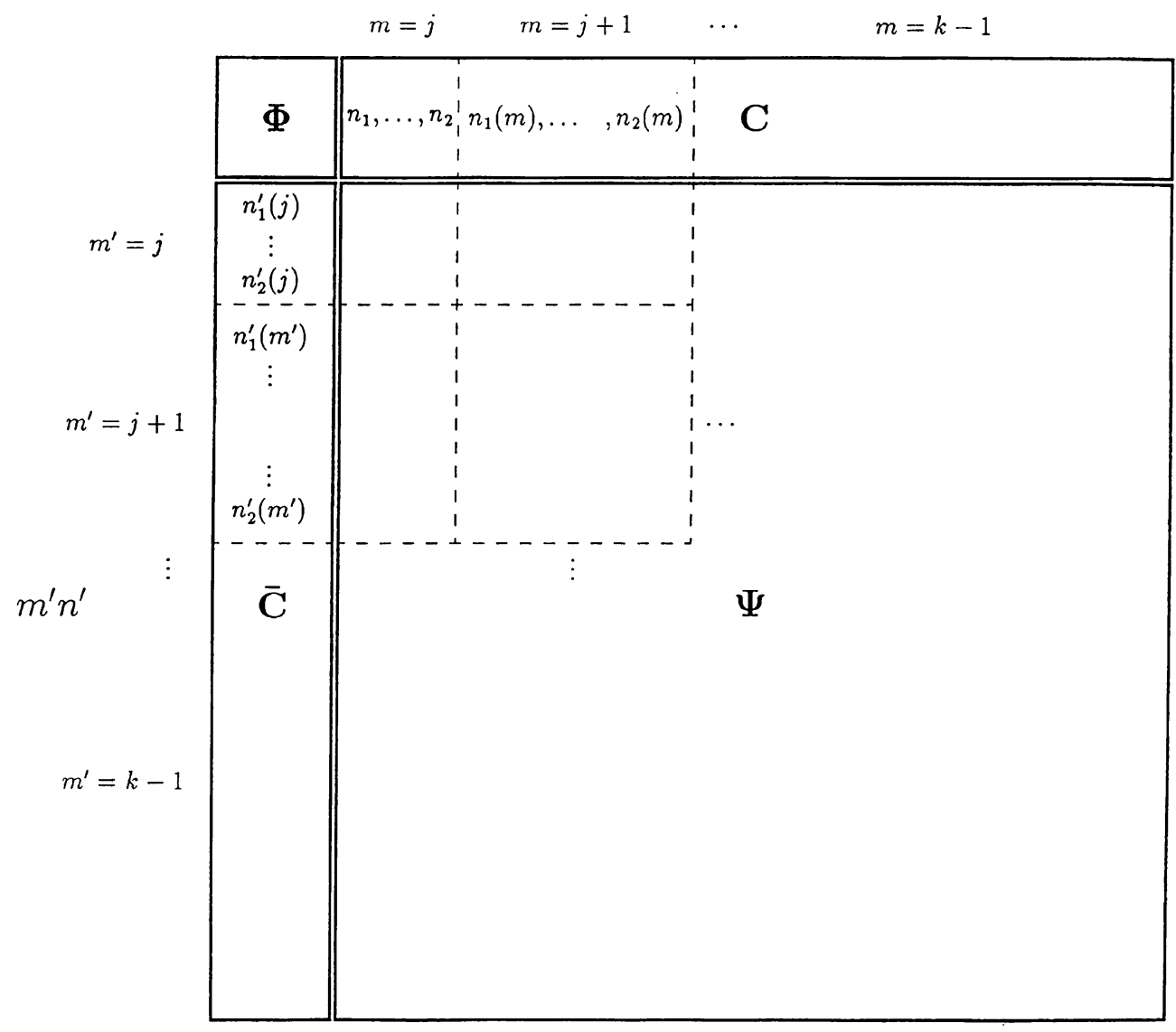

FIG. 3. Schematic of the matrix operators

formulation smooth. From the lower half of (3.8), the response detail $\vec{d}$ can be expressed in terms of the response smooth $\vec{s}$ and the excitation detail $\vec{E}$. When this is substituted into the upper half of Eq. (3.8) we get a formulation governing $\vec{s}$ - the formulation smooth,

$$
\left[\mathbf{I}-\boldsymbol{\Phi}-\mathbf{C}(\mathbf{I}-\mathbf{\Psi})^{-1} \overline{\mathbf{C}}\right] \vec{s}=\vec{e}+\mathbf{C}(\mathbf{I}-\Psi)^{-1} \vec{E} .
$$

It has been pointed out in [1] that $\Phi$ can be interpreted as a "smoothed version" of the operator $\mathbf{L}$, a representation of the latter by a straightforward discretization on a reference grid separation $2^{-j}$. Thus $\mathbf{C}(\mathbf{I}-\boldsymbol{\Psi})^{-1} \overline{\mathbf{C}}$ has been interpreted as an effective material operator, an operator describing the across-scales coupling due to the presence of a microstructure. This operator, as well as the smoothed operator $\boldsymbol{\Phi}$, was studied in $[1,2]$ for the problem of fluid-loaded thin elastic plates with nonstationary mass density or stiffness microstructure. From the definitions in (3.9), it is evident that the matrix entries and the effective material operator properties depend on the operator $\mathbf{L}$, or more specifically, on the background problem kernel $G(x)$ and on the system heterogeneity $h(x)$ (see (3.1)-(3.2b)). The next section summarizes previous results and establishes a 
relation between the macro- and micro-responses that will be used in subsequent derivations.

4. Properties of the formulation smooth. The manner in which the microstructure affects the effective material operator $\mathbf{C}(\mathbf{I}-\mathbf{\Psi})^{-1} \overline{\mathbf{C}}$ in (3.10) has been investigated in $[1,2]$ for the case of a heterogeneity with widely separated micro- and macro-scales, pertaining to a thin, elastic, fluid-loaded plate. Since these results play a pivotal role in the present theory, we shall briefly summarize them in the following subsection.

4.1. System characterization and scales hierarchy. Two types of length-scales are defined by the physical system. The first applies to $t^{\mathrm{l}} \mathrm{e}$ variation of $G(x)$, induced by the dynamics of the system. We refer to this as tine wavelength $(\lambda)$ scale and to the associated resolution as $m_{\lambda}$. The possible irregularity of $G(x)$ at the origin may introduce additional variability over a relatively wide range of length-scales. This irregularity has an essential role in the theory and it will be discussed further. The second type of length-scale is due to the system heterogeneity and applies to the variations of $q(x)$. These variations can range from the macroscale $(\geq \lambda)$ to the microscale $(\ll \lambda)$. The former and the latter are associated with the resolutions $m_{a}$ and $m_{i}$, respectively, such that $2^{-m_{a}} \geq \lambda$ and $2^{-m_{i}} \ll \lambda$. Finally, the reference, or smoothing, scale should be carefully selected. Details of scale $\lambda$ must be adequately described, whereas microscale details can be averaged. The following hierarchy of resolutions and scales applies:

$$
m_{a} \leq m_{\lambda}<j<m_{i} \Leftrightarrow 2^{-m_{a}} \geq 2^{-m_{\lambda}} \approx \lambda \gg 2^{-j} \gg 2^{-m_{i}} .
$$

Essential to the present study is the concept of a "scatterer", as a localized region of $m i$ croscale heterogeneity that is confined to a domain in space measured on the macroscale. Also essential is the existence of a "gap" in the scales for observing heterogeneity. Specifically, the system heterogeneity is not to possess any component in the range of scales $\left(2^{-m_{a}-1}, 2^{-m_{i}+1}\right)$. We term this case a two-scale (macro/micro) variation. When referred to the $j$ scale, i.e., the chosen smoothing scale, the macroscale variation is the smooth component and the microscale variation is the detail component. We write

$$
h(x)=h^{s}(x)+h^{d}(x),
$$

where

$$
h^{s}(x)=\sum_{n} a_{n} \phi_{m_{a} n}(x)
$$

is expressible as a synthesis of scaling functions and

$$
h^{d}(x)=\sum_{n} b_{n} \psi_{m_{i}-1 n}(x)
$$

as a synthesis of wavelets. The index $n$, in both of these equations, spans a region with physical dimensions described on the macroscale. Note that $h^{s}(x)$ and $h^{d}(x)$ are mutually orthogonal, and the associated scales are widely separated. The synthesis in $(4.2 \mathrm{a})-(4.2 \mathrm{~b})$ identifies the spatial averages of $h(x)$ over neighborhoods in the order of the macroscale $2^{-m_{a}}$ with $h^{s}$, and further implies that spatial averages of the detail component $h^{d}=h-h^{s}$ vanish if performed over neighborhoods larger than the microscale 
$2^{-m_{i}}$. Denoting by $\|\cdot\|_{2}$ the $L_{2}$-norm, we shall assume throughout the rest of this work that

$$
\left\|h^{d}(x)\right\|_{2}=O\left(\left\|h^{s}(x)\right\|_{2}\right)=O(1)
$$

which is equivalent to

$$
\sum_{n}\left|b_{n}\right|^{2}=O\left(\sum_{n}\left|a_{n}\right|^{2}\right)=O(1) .
$$

In other words, the energy associated with the microstructure is of the order of that associated with the macrostructure, and both are beyond the perturbative regime.

4.2. The response components $u^{s}, u^{d}$, and the microstructure signature.

4.2.1. Smooth $G(x)$. We assume that the integral equation kernel function $G(x)$ is regular at the origin (that is, it varies on the scale of $\lambda$ for all $x$ ). Then, an estimate of the matrix elements in Eqs. (3.9) can be obtained by invoking Eqs. (2.5a)-(2.6) and the scale hierarchy characterization (4.1). We get (see [1])

$$
\begin{aligned}
\Phi_{n^{\prime} . n} & \simeq \overline{\phi_{j n^{\prime}}^{f}\left(x_{j n}\right)}\left\langle h, \phi_{j n}\right\rangle, \\
\Psi_{m^{\prime} n^{\prime} \cdot m n} & \simeq \overline{\psi_{m^{\prime} n^{\prime}}^{f}\left(x_{m n}+2^{-m-1}\right)}\left\langle h, \psi_{m n}\right\rangle, \\
C_{n^{\prime} \cdot m n} & \simeq \overline{\phi_{j n^{\prime}}^{f}\left(x_{m n}+2^{-m-1}\right)}\left\langle h, \psi_{m n}\right\rangle, \\
\bar{C}_{m^{\prime} n^{\prime}, n} & \simeq \overline{\psi_{m^{\prime} n^{\prime}}^{f}\left(x_{j n}\right)}\left\langle h, \phi_{j n}\right\rangle,
\end{aligned}
$$

where the overbars on the right-hand sides denote complex conjugate, and $\phi^{f}, \psi^{f}$ are the "fields" obtained by the adjoint of the background operator $\mathbf{L}_{b}$,

$$
\begin{aligned}
\phi_{j n}^{f}(y) & =\mathbf{L}_{b}^{*} \phi_{j n} \simeq 2^{-j / 2} \overline{G\left(x_{j n}-y\right)}, \\
\psi_{m n}^{f}(y) & =\mathbf{L}_{b}^{*} \psi_{m n} \simeq \beta 2^{-m(\Lambda+1 / 2)} \overline{G_{M I}\left(x_{m n}+2^{-m-1}-y\right)} .
\end{aligned}
$$

With the articulated conditions on $G$ and the results in Eqs. (2.5b)-(2.6) it follows that $\left|\psi_{m n}^{f}(y)\right| \ll 1$. Together with (4.3a)-(4.3b) it induces the norm relations:

$$
\begin{aligned}
& \|\Psi\| \ll 1, \\
& \|\overline{\mathbf{C}}\| \ll 1, \\
& \|\mathbf{C}\| \leq O(1),
\end{aligned}
$$

where $\|\cdot\|$ denotes the matrix norm induced by the Euclidean vector norm. These estimates are derived in the appendix. It should be emphasized that they are valid only for smooth $G(x)$ and for the scatterers characterized by (4.1) and the discussion thereafter. With (4.6a)-(4.6c) one obtains for the effective material operator $\| \mathbf{C}(\mathbf{I}-$ $\boldsymbol{\Psi})^{-1} \overline{\mathbf{C}} \| \ll 1$. The conclusion that, under (4.3a) and the smoothness condition on $G$, the macroscale response practically bares no footprint of the microscale heterogeneity, follows directly from this last result (see [1]). Futhermore, this result establishes that a new formulation, governing the macroscale response component $u^{s}(x)$, is readily obtained from (3.1)-(3.2b),

$$
u^{s}(x)=u_{0}(x)+\mathbf{L}_{b} h^{s} u^{s}
$$


where $\mathbf{L}_{b}$ is the background operator defined in $(3.2 \mathrm{~b}), u_{0}(x)$ is the excitation term, assumed here to vary on the macroscale only, and $h^{s}(x)$ is the locally averaged (smooth component) of the system heterogeneity (see (4.2)-(4.2b) and discussion thereafter). Equation (4.7) identifies $h^{s}(x)$ as a local effective property of the system heterogeneity $h(x)$.

The inequalities in (4.6a)-(4.6c) can be used to derive an estimate on the relation between $u^{s}$ and $u^{d}$. From the multiscale resolved formulation (3.8),

$$
(\mathbf{I}-\mathbf{\Psi}) \vec{d}=\overline{\mathbf{C}} \vec{s}+\vec{E} .
$$

Using (4.6a), and subsequently applying the triangle and operator norm inequalities, we get

$$
\|\vec{d}\| \leq\|\overline{\mathbf{C}}\|\|\vec{s}\|+\|\vec{E}\|
$$

where $\|u\|$ denotes the Euclidean vector norm if $u$ is a vector, or the matrix norm induced by the Euclidean vector norm if $u$ is a matrix. In cases where the excitation term $u_{0}$ is described on the macroscale only (i.e., $\|\vec{E}\|=0$ ) we get (with (4.6b))

$$
\|\vec{d}\| \ll\|\vec{s}\|
$$

or, denoting by $\|\cdot\|_{2}$ the $L_{2}$-norm (taking over the support of the heterogeneity),

$$
\left\|u^{d}\right\|_{2} \ll\left\|u^{s}\right\|_{2} .
$$

The last result has an important role in the construction of effective constitutive relations pertaining to $1 \mathrm{D}$ plate stiffness variations.

4.2.2. Singular $G(x)$. In cases where the integral equation background kernel $G(x)$ is singular at the origin, the regularity assumptions, leading to (4.6a)-(4.6c), cannot be used. Thus, the inequality $\left\|\mathbf{C}(\mathbf{I}-\mathbf{\Psi})^{-1} \overline{\mathbf{C}}\right\| \ll 1$ does not hold in general. The immediate consequence is that a microstructure can have a significant effect on the macroresponse. This has been investigated and demonstrated in [2] for the specific case of a fluid-loaded thin elastic plane with a 1D stiffness variation.

\section{Implementation to composite materials mechanics.}

5.1. Statement of the problem. We shall be concerned with the flexural velocity response of a thin elastic plate lying in the $z=0$ plane, due to a time harmonic $\left(e^{i \omega t}\right)$ $y$-independent excitation. A surface mass density heterogeneity and a stiffness heterogeneity are introduced, respectively, by the $y$-independent distributions

$$
\begin{aligned}
& m(x)=m_{0}[1+\rho(x)], \\
& Q(x)=Q_{0}[1+q(x)],
\end{aligned}
$$

where $\rho(x)$ and $q(x)$ are normalized, dimensionless distributions expressing the local variations with respect to the background values $m_{0}$ and $Q_{0}$. The flexural wavenumber associated with $m_{0}$ and $Q_{0}$ is given by $k_{f_{0}}=\left(m_{0} \omega^{2} / Q_{0}\right)^{1 / 4}$. With the dimensionless length coordinate $x \mapsto k_{f_{0}} x$, the equation of motion governing the flexural velocity response $v(x)$ due to a $y$-independent forcing $F(x)$ is

$$
\frac{d^{2}}{d x^{2}}\left\{[1+q(x)] \frac{d^{2}}{d x^{2}} v(x)\right\}-[1+\rho(x)] v(x)=-\frac{i}{\omega m_{0}} F(x) .
$$




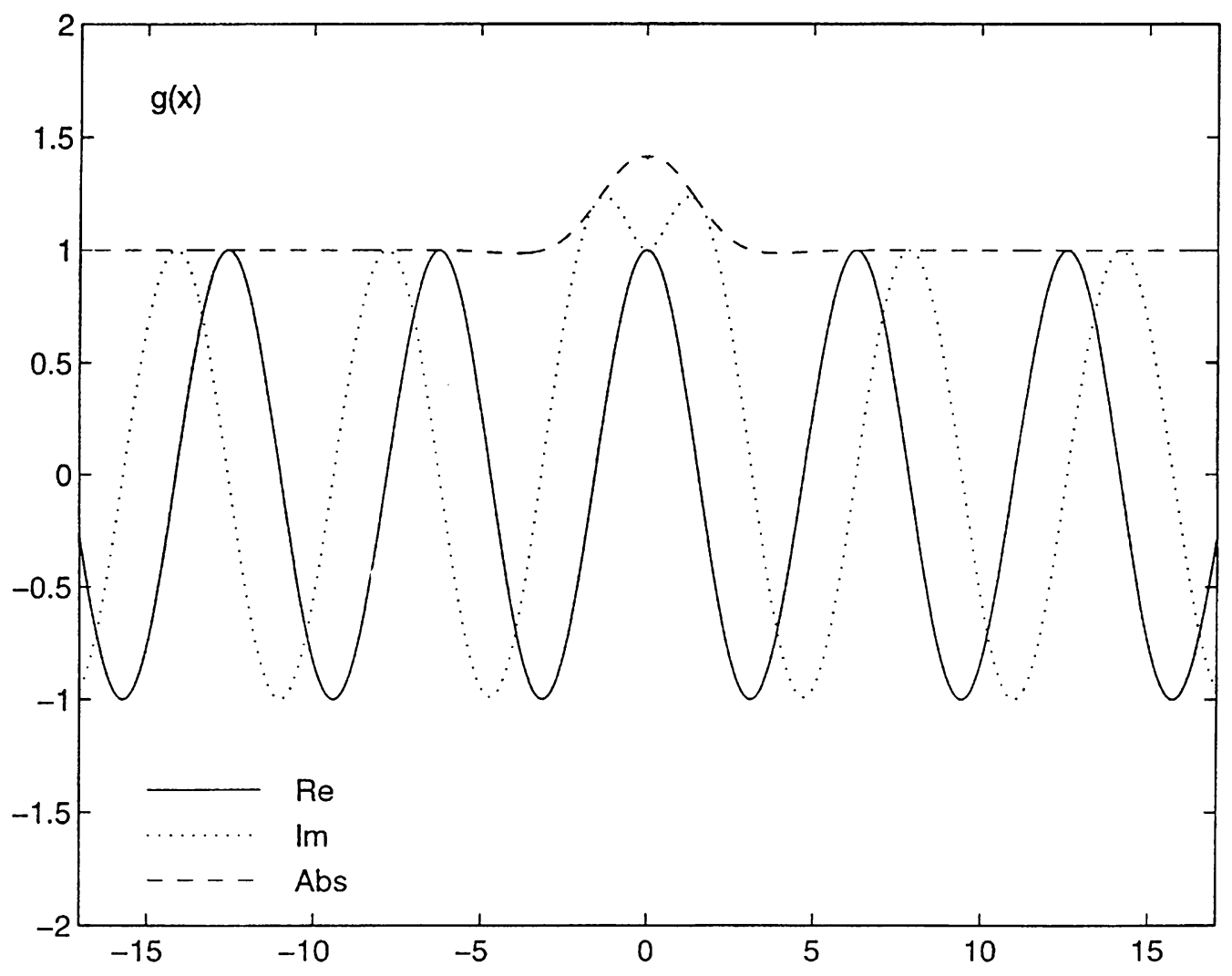

FIG. 4. The homogeneous plate Green function $g(x)$

The background plate Green function (i.e., the response of the homogeneous plate $q(x)=$ $\rho(x)=0$ to a line forcing $F(x)=\delta(x))$ is given by

$$
g(x)=\frac{1}{4 \omega m_{0}}\left(e^{i|x|}+i e^{-|x|}\right) .
$$

This response is shown in Fig. 4. It is a smooth function, described essentially on the scale of $2 \pi$. It has a third derivative discontinuity at $x=0$ and a variation scale of $\lambda=2 \pi$ away from the origin. Consistent with (4.1) the macro, reference, and micro resolutions are defined as $m_{a}<1, j=0$, and $m_{i}=2$, respectively. These values correspond to the wavelength $\lambda$, a reference scale of about $\lambda / 6$, and a microscale in the order of $\lambda / 24$. Figures $5(\mathrm{a})$ and $5(\mathrm{~b})$ show two different manifestations of microstructures, namely $h_{1}^{d}(x)$ and $h_{2}^{d}(x)$, synthesized via $(4.2 \mathrm{~b})$ with the articulated definitions of scales. For each manifestation, the coefficients $b_{n}$ were synthesized by a random number generator. Figure 5(c) shows an example of a macrostructure $h^{s}(x)$. Evidently, $h_{1.2}^{d}(x)$ vary on a length-scale much smaller than the (normalized) wavelength $\lambda=2 \pi$, and the $h^{s}(x)$ variation length-scale is in the order of $\lambda$. 


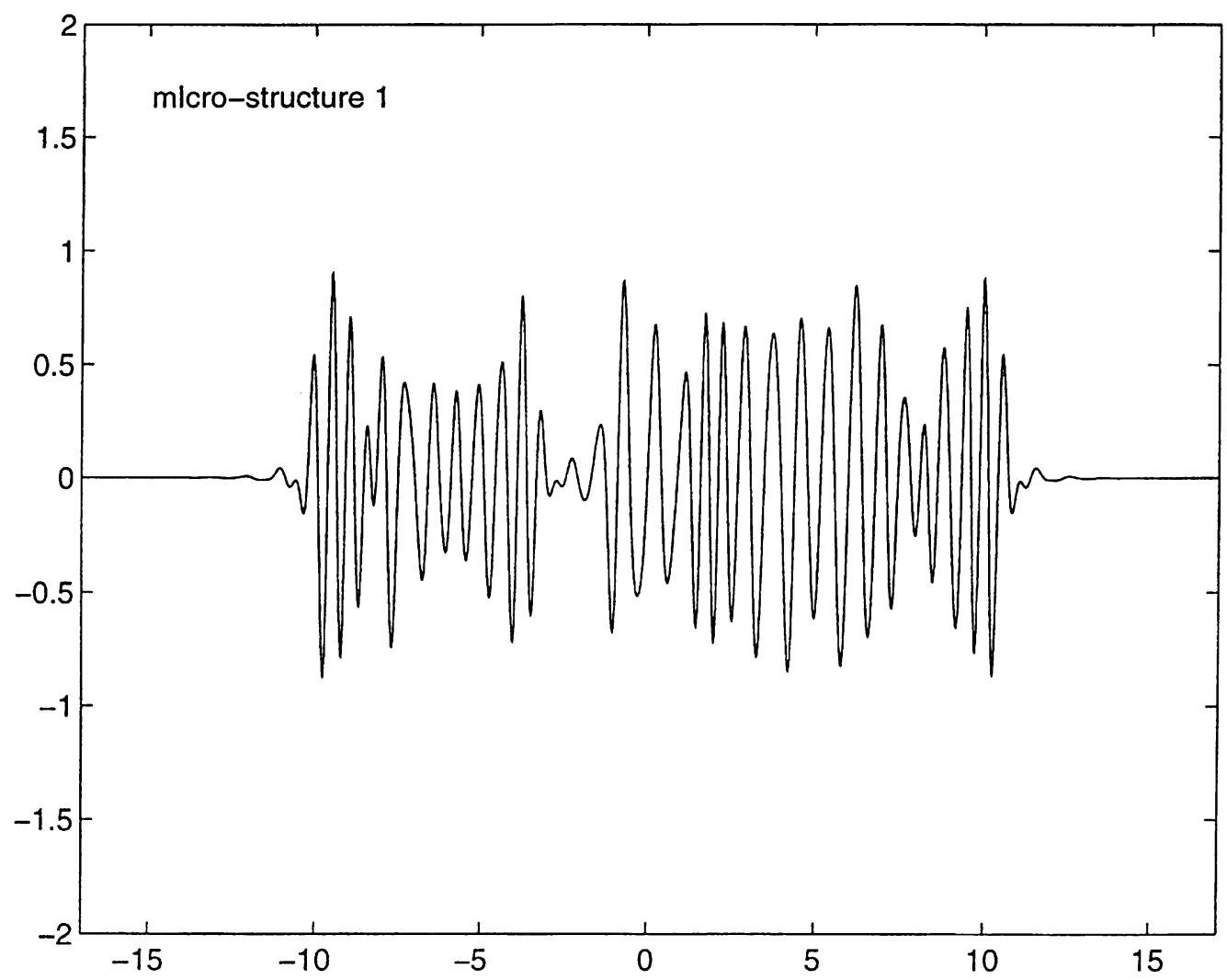

FIG. 5(a). Examples of heterogeneity. The microstructure $h_{1}^{d}(x)$

In the next sections we shall examine the plate response associated with mass or stiffness complex scatterers - examples of which are given by $h_{1.2}^{d}(x)$, or by linear combinations of the latter with $h^{s}(x)$. Specifically, we shall show that

1. Microscale mass heterogeneity with macroscale outer dimension has no footprint in the macroscale response.

2. Microscale stiffness heterogeneity with macroscale outer dimension can significantly affect the macroscale response.

3. It is possible to synthesize classes of different stiffness complex scatterers such that the macroscale response associated with each of the members of the class is the same.

These results are first obtained by using theoretical considerations and then demonstrated numerically. As seen in the next two subsections, points 1 and 2 above follow directly from the analysis of Sec. 4.2. Point 3 needs more detailed analysis and is treated in a separate section. 


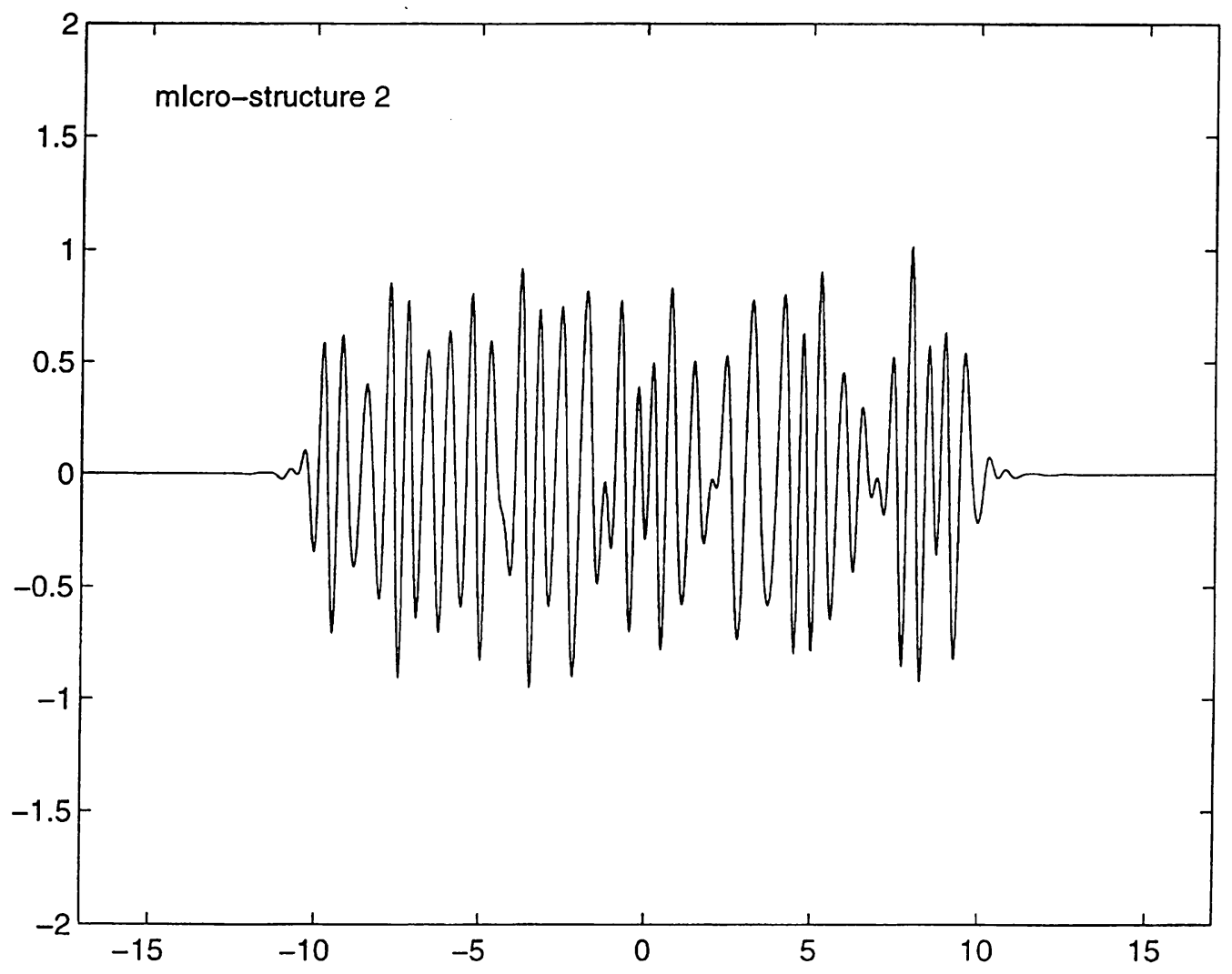

FIG. 5(b). Examples of heterogeneity. The microstructure $h_{2}^{d}(x)$

5.2. Mass variation. Here we examine the case of mass density variation and constant stiffness. Thus, $q(x)=0$ and the equation of motion (5.2) can be rewritten as

$$
\frac{d^{4}}{d x^{4}} v(x)-v(x)=\frac{-i}{\omega m_{0}}\left[F(x)+i \omega m_{0} \rho(x) v(x)\right] .
$$

By expressing the solution $v(x)$ as a convolution of $g(x)$ with the forcing term $F(x)+$ $i \omega m_{0} \rho(x) v(x)$, the Fredholm integral equation formulation (3.1)-(3.2b) is obtained with

$$
\begin{aligned}
u(x) & =v(x), \\
h(x) & =\rho(x), \\
G(x) & =i \omega m_{0} g(x), \\
u_{0}(x) & =v_{0}(x)=\int g\left(x-x^{\prime}\right) F\left(x^{\prime}\right) d x^{\prime},
\end{aligned}
$$

where $g(x)$ is defined in (5.3). It is seen from (5.5c) that the kernel $G(x)$ associated with the integral operator is smooth. The results of Sec. 4.2 .1 can be cited directly: (i) microscale mass variation has no footprint in the macroscale response, the latter is governed now by the formulation (4.7), and (ii) if $v_{0}$ in $(5.5 \mathrm{~d})$ is described on the 


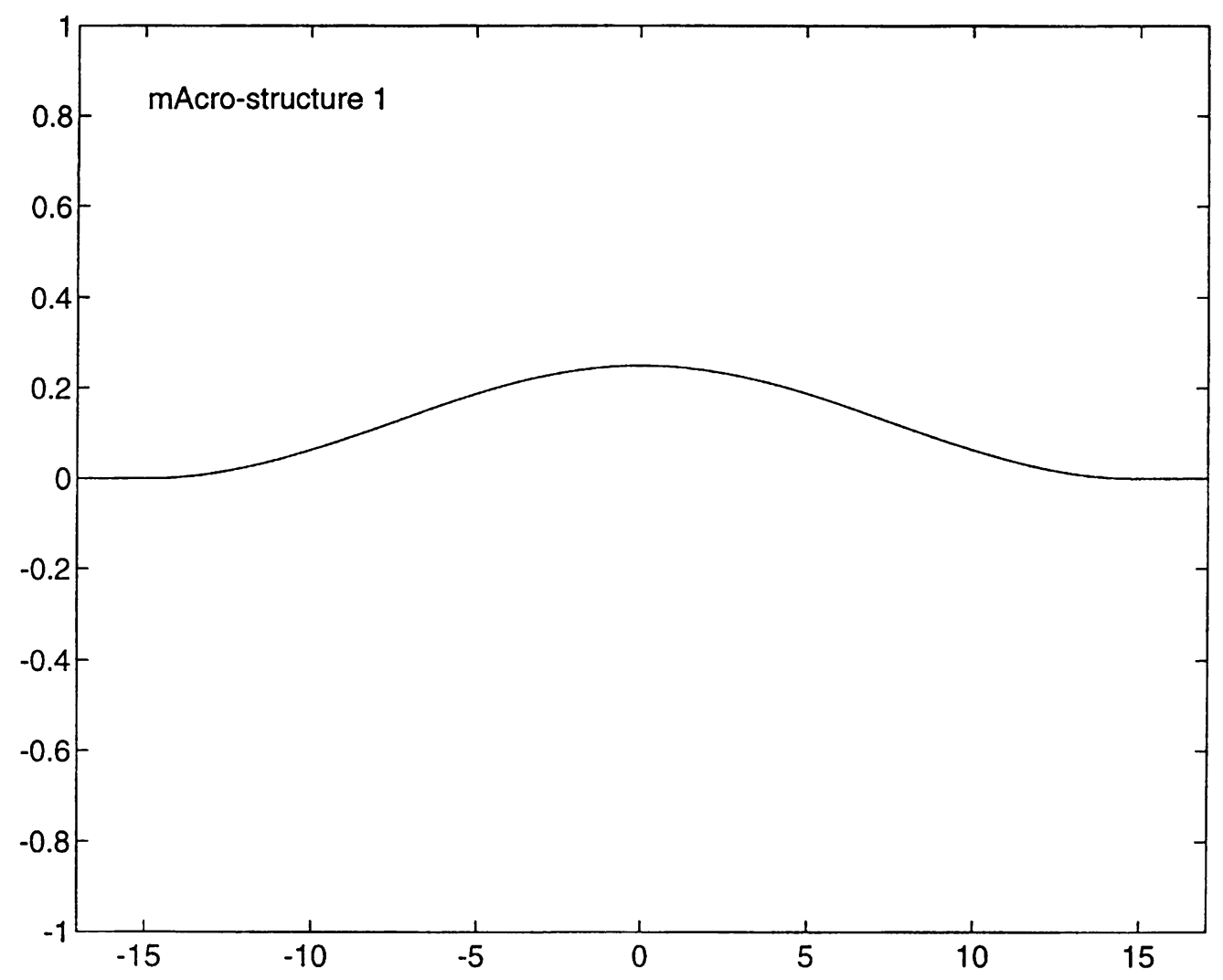

FIG. 5(c). Examples of heterogeneity. The macrostructure $h^{s}(x)$

macroscale only, then a relation similar to (4.10b) applies for $v(x)$. Note that this last condition holds for every "reasonable" $F(x)$ - a line forcing, for example.

A numerical simulation demonstrates these results. We have computed the plate responses, associated with the following mass heterogeneities:

$$
\begin{aligned}
& \rho_{1}(x)=h_{1}^{d}(x), \\
& \rho_{2}(x)=h^{s}(x), \\
& \rho_{3}(x)=h^{s}(x)+h_{1}^{d}(x),
\end{aligned}
$$

where $h_{1}^{d}(x)$ and $h^{s}(x)$ are shown in Fig. 5. Thus, $\rho_{1}(x)$ possesses microscale variations only, and $\rho_{3}(x)$ possesses both a microstructure $\left(h_{1}^{d}(x)\right)$ and a macrostructure $\left(h^{s}(x)\right)$. The forcing term is given by $F(x)=\phi(x-15)$, which corresponds to a somewhat smoothed line forcing situated at $x^{\prime}=15$, and is shown in Fig. 6, drawn to scale together with the corresponding background solution $(5.5 \mathrm{~d})$. Figure 7 shows the complete response that corresponds to the heterogeneity $\rho_{1}(x)$. A comparison with the background solution (Fig. 6) shows that $\rho_{1}(x)$, which consists of microscale variations only, has no footprint on the response. Figure 8 compares the macroscale responses associated with $\rho_{2}(x)=h^{s}(x)$ and $\rho_{3}(x)=h^{s}(x)+h_{1}^{d}(x)$. These mass density distributions differ only 


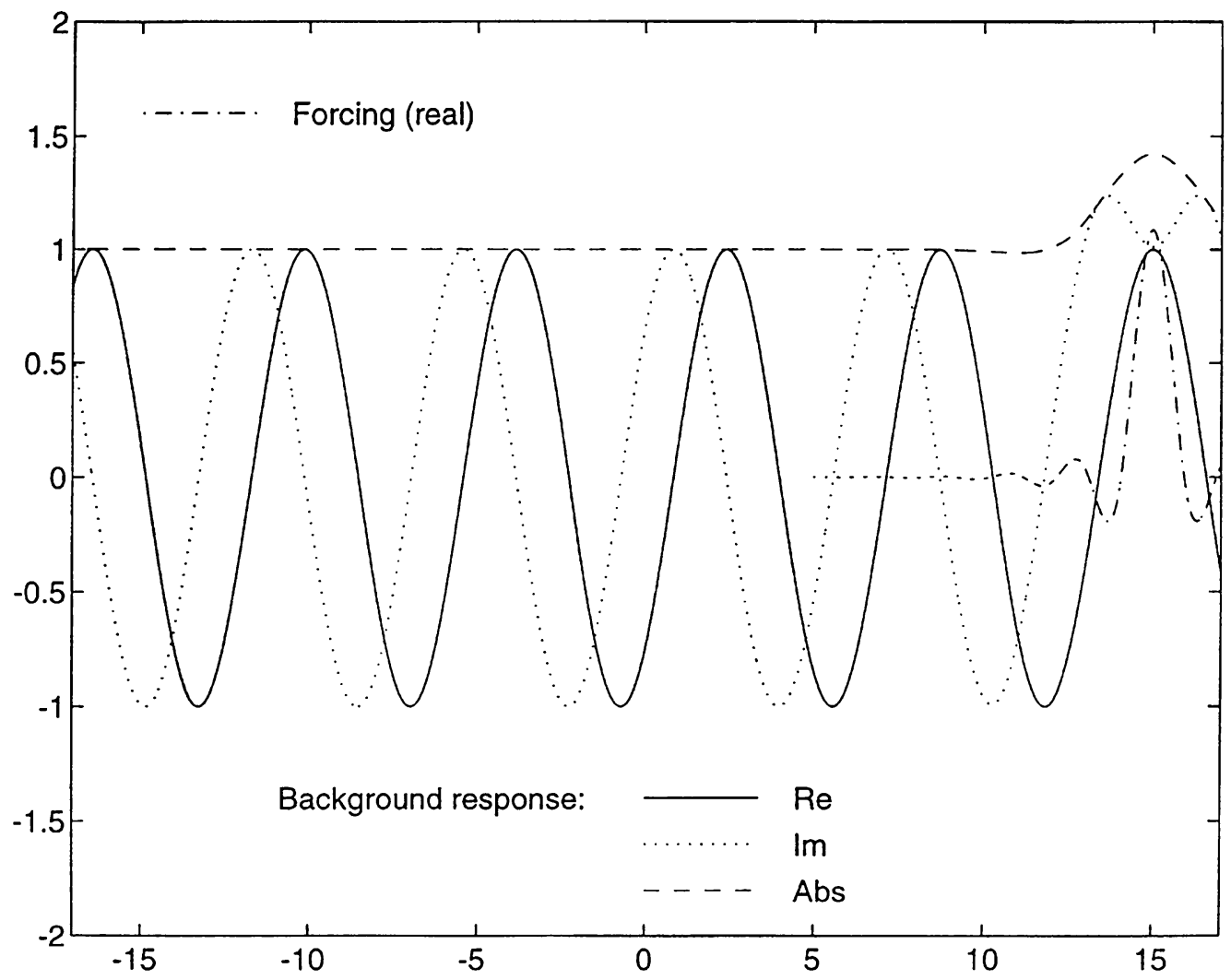

Fig. 6. The forcing term $F(x)$ and the background system response for the mass heterogeneity problem

by their microscale components ( 0 and $h_{1}^{d}(x)$, respectively). The macroscale responses are indistinguishable within the graphic resolution. Finally, Fig. 9 depicts the microscale component of the plate response - the inequality of $(4.10 \mathrm{~b})$ is evident.

5.3. Stiffness variations. If we set $\rho(x)=0, q(x) \neq 0$, the equation of motion (5.2) can be rewritten as

$$
\frac{d^{4}}{d x^{4}} v(x)-v(x)=\frac{-i}{\omega m_{0}}\left[F(x)-i \omega m_{0} \frac{d^{2}}{d x^{2}}\left\{q(x) \frac{d^{2}}{d x^{2}} v(x)\right\}\right] .
$$

By expressing the solution $v(x)$ as a convolution of $g(x)$ with the right-hand side of Eq. (5.7), and taking the second derivative of the resulting formulation with respect to $x$, we get the integro-differential equation

$$
u(x)=u_{0}(x)-i \omega m_{0} \int g^{\prime \prime}(x-y)[q(y) u(y)]^{\prime \prime} d y
$$

where a prime denotes a derivative with respect to the argument and

$$
\begin{aligned}
u(x) & =v^{\prime \prime}(x), \\
u_{0}(x) & =\int g^{\prime \prime}(x-y) F(y) d y .
\end{aligned}
$$




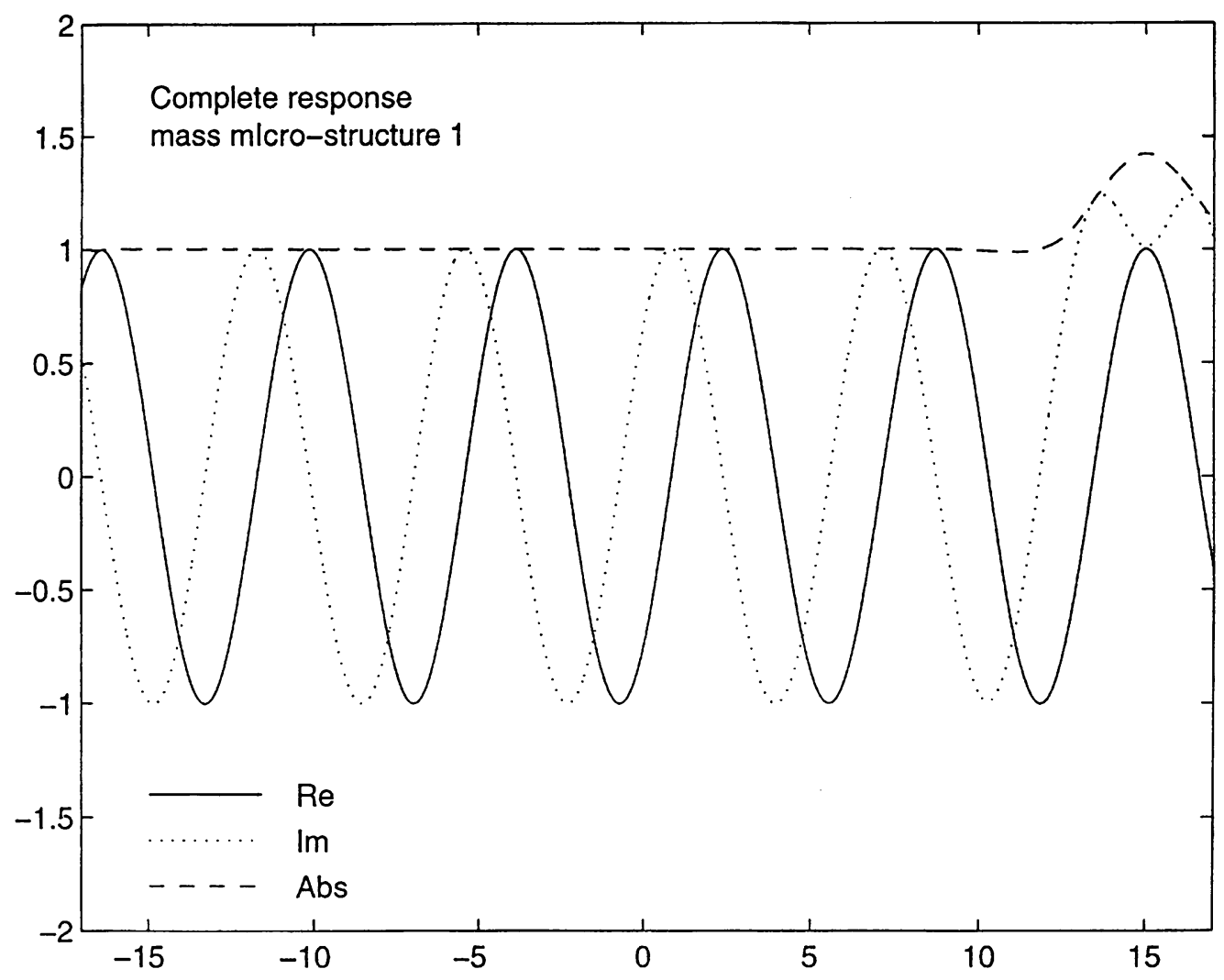

FIG. 7. The response associated with $\rho_{1}(x)=h_{1}^{d}(x)$ (a microstructure only)

Note that $u(x)$ can be interpreted as the reciprocal of the plate local radii of curvature. Integrating (5.8) by parts twice, and assuming that $q(x)$ and all its derivatives vanish at infinity, we get the Fredholm integral equation formulation (3.1)-(3.2b) with $u, u_{0}$ defined in (5.8a)-(5.8b) and with

$$
\begin{aligned}
h(x) & =q(x), \\
G(x) & =-i \omega m_{0} g(x)-\delta(x),
\end{aligned}
$$

where $\delta(x)$ is the Dirac delta function and $g(x)$ is defined in (5.3). Evidently, the kernel function $G(x)$ of the integral operator is now highly singular at the origin. Referring to Sec. 4.2 .2 , the immediate consequence is that a stiffness microstructure can have a significant effect on the macroscale response. 


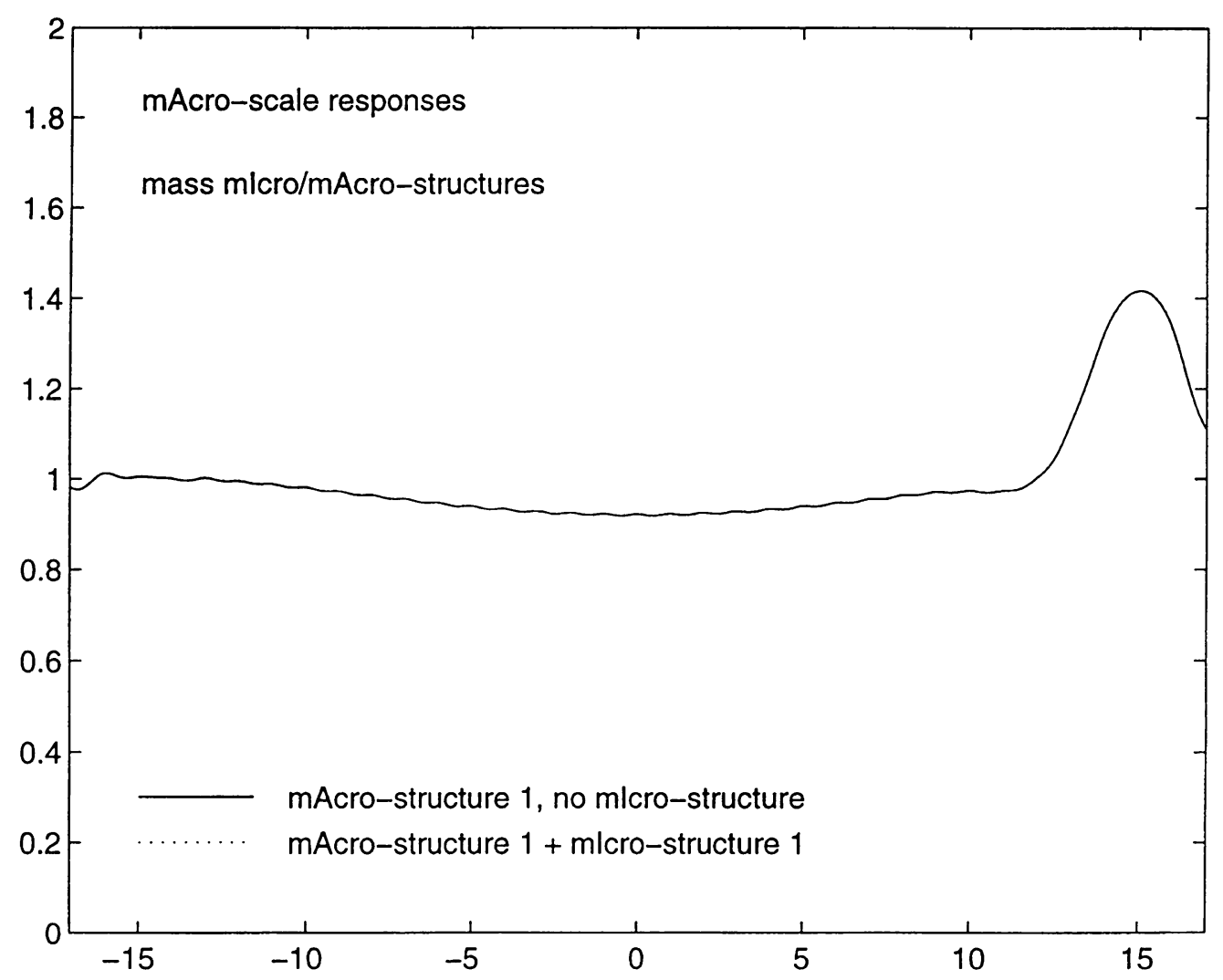

FIG. 8. Magnitude of the macroscale component of the plate responses, $u^{s}(x)$, associated with $\rho_{2}(x)$ (solid line), and $\rho_{3}(x)$ (dot line). To the resolution of this graph the two solutions cannot be distinguished.

A numerical example demonstrates these results. We have computed the response of a plate with the following stiffness heterogeneities:

$$
\begin{aligned}
& q_{1}(x)=h_{1}^{d}(x), \\
& q_{2}(x)=h_{2}^{d}(x), \\
& q_{3}(x)=h^{s}(x), \\
& q_{4}(x)=h^{s}(x)+h_{1}^{d}(x),
\end{aligned}
$$

where $h_{1,2}^{d}(x)$ and $h^{s}(x)$ are shown in Fig. 5. Note that $q_{1}$ and $q_{2}$ possess microscale variations only, $q_{3}$ possesses macroscale variations, whereas $q_{4}$ possesses both, with the macroscale component identical to that of $q_{3}(x)$. The forcing term is again given by $F(x)=\phi(x-15)$, and is shown in Fig. 10 drawn to scale together with the corresponding background solution (5.8b). Figure 11 compares the macroscale response component $u^{s}$ associated with $q_{1}$ to that associated with $q_{2}$. The difference is evident and it is also evident that both deviate significantly from the background response. Recall that $q_{1.2}$ possess microscale variations only-these results confirm our assertion that microscale 


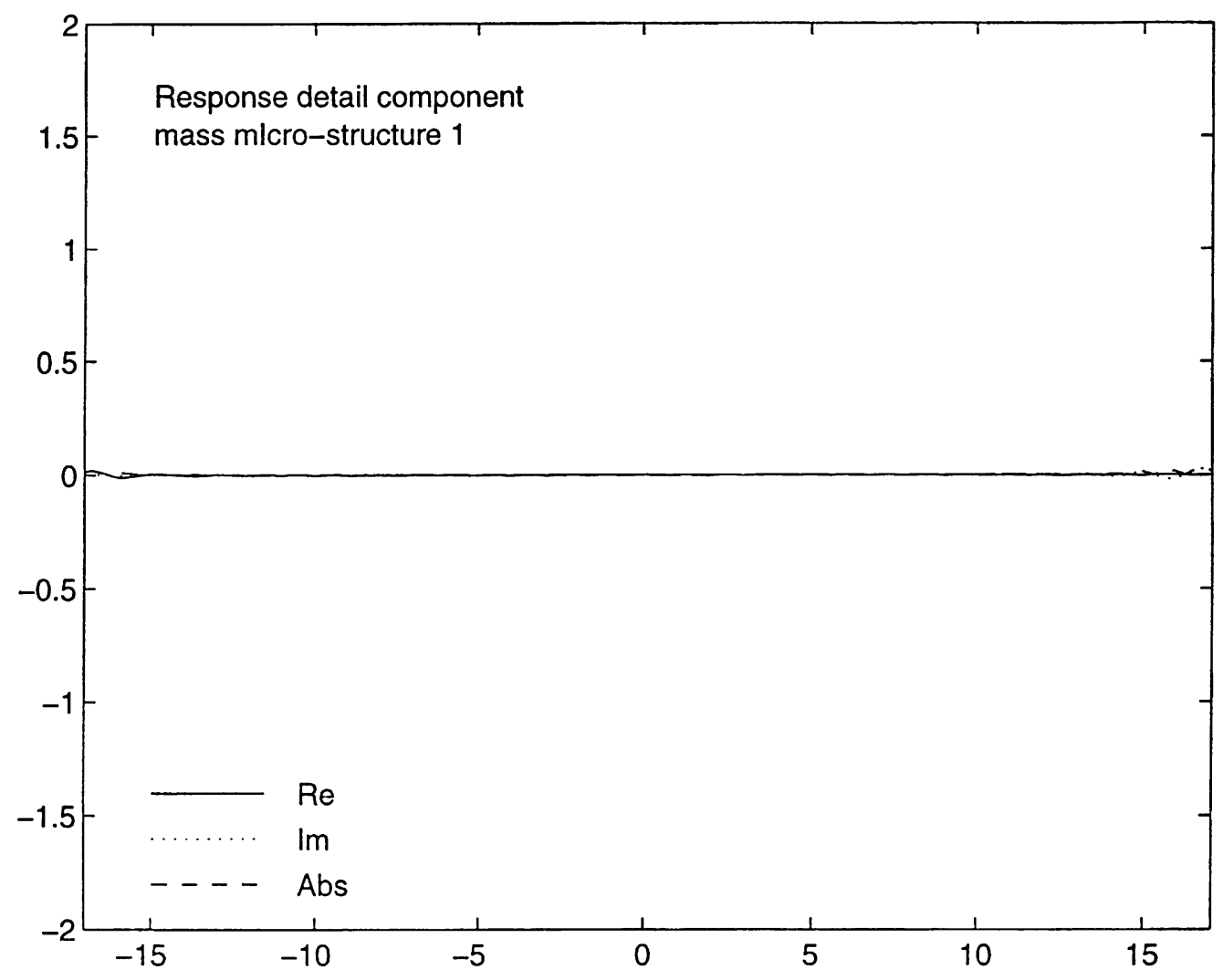

FIG. 9. The microscale component of the plate response, $u^{d}$, associated with $\rho_{1}(x)=h_{1}^{d}(x)$

stiffness variation significantly affects macroscale response. This holds also in the presence of a macroscale variation. Figure 12 shows a comparison of $u^{s}$ associated with $q_{3}$ to that associated with $q_{4}$. Again, the difference is evident.

6. The effective constitutive relations. By substituting $(5.9 \mathrm{~b})$ into the integral equation formulation (3.1)-(3.1a), performing the integration over the $\delta\left(x-x^{\prime}\right) q\left(x^{\prime}\right) u\left(x^{\prime}\right)$ term, and moving the result to the left-hand side, we get the integral equation

$$
u(x)[1+q(x)]=u_{0}(x)-i \omega m_{0} \int g(x-y) q(y) u(y) d y .
$$

In this equation, $Q(x)=1+q(x)$ is the local bending stiffness of the plate; the plate bending moment, $T(x)$, is related to $u(x)$ via the local constitutive relation

$$
T(x)=u(x) Q(x)=u(x)[1+q(x)] .
$$

The last results suggest a new integral equation, written for the bending moment $T(x)$,

$$
T(x)=T_{0}(x)+\Gamma_{b} r T
$$




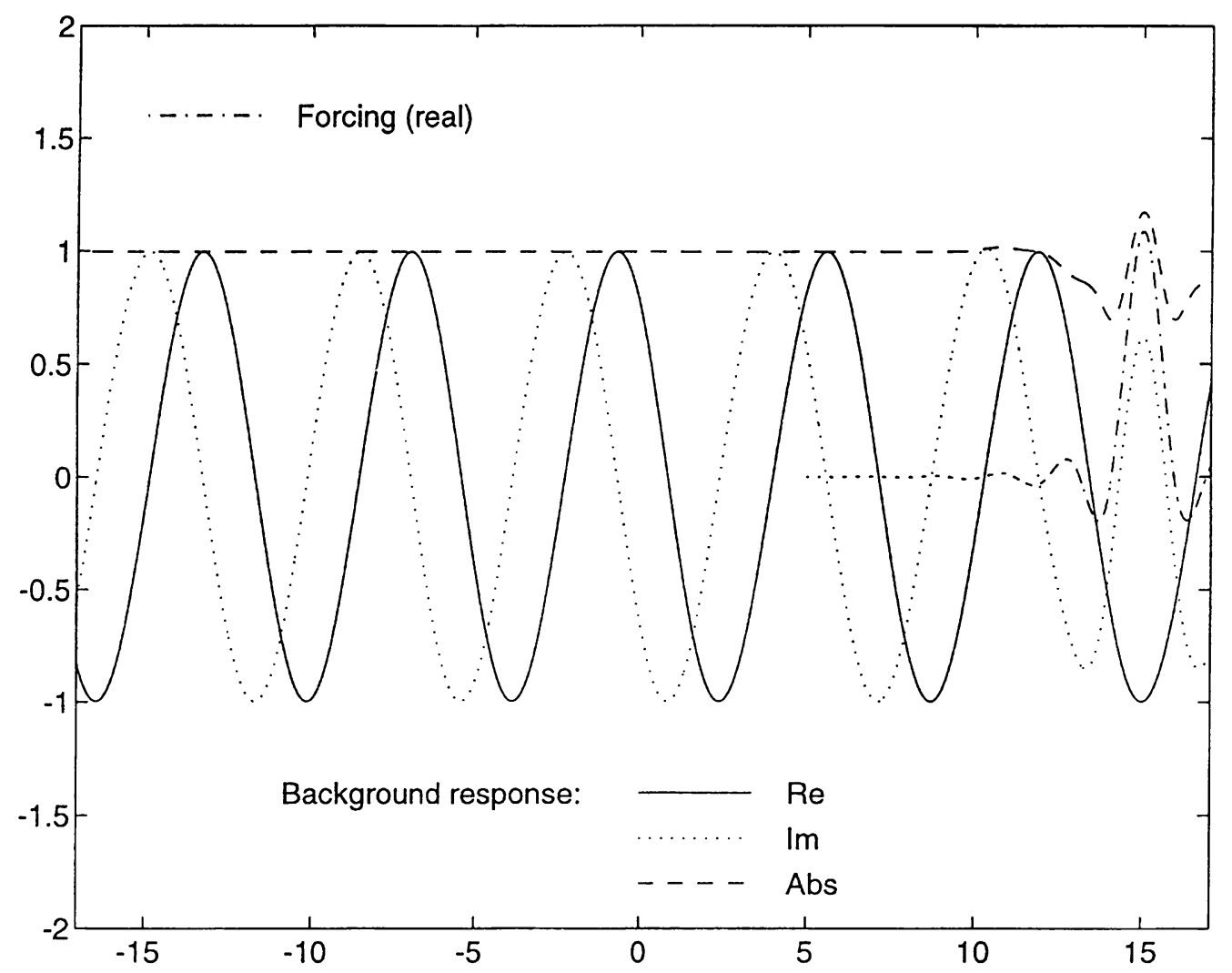

Fig. 10. The forcing term $F(x)$ and the background system response for the stiffness heterogeneity problem

with $T_{0}(x)$ as the background bending moment (corresponds to $q(x)=0$ ),

$$
T_{0}(x)=u_{0}(x) .
$$

$\boldsymbol{\Gamma}_{b}$ is an integral operator associated with the smooth kernel $g(x)$,

$$
\Gamma_{b} f=-i \omega m_{0} \int g(x-y) f(y) d y
$$

and $r=r(x)$ is a heterogeneity measure related to the stiffness variability,

$$
r(x)=q(x) /[1+q(x)] .
$$

Thus, the bending moment $T(x)$ is governed by a second-kind Fredholm integral equation, a formulation completely analogous to $(3.1)-(3.2 \mathrm{~b})$ with a smooth background kernel function. The forcing term $F(x)$ is assumed to possess variations on the macroscale only. The spectrum of $g(x)$, namely $\tilde{g}(\xi)$, behaves like $\xi^{-4}$ for $\xi>2 \pi / \lambda$ (see [1]) -rendering negligible the detail component of the excitation term $T_{0}=u_{0}$. Hence, the results of Sec. 4.2.1 directly apply:

$$
T^{s}(x)=T_{0}(x)+\Gamma_{b} r^{s} T^{s},
$$




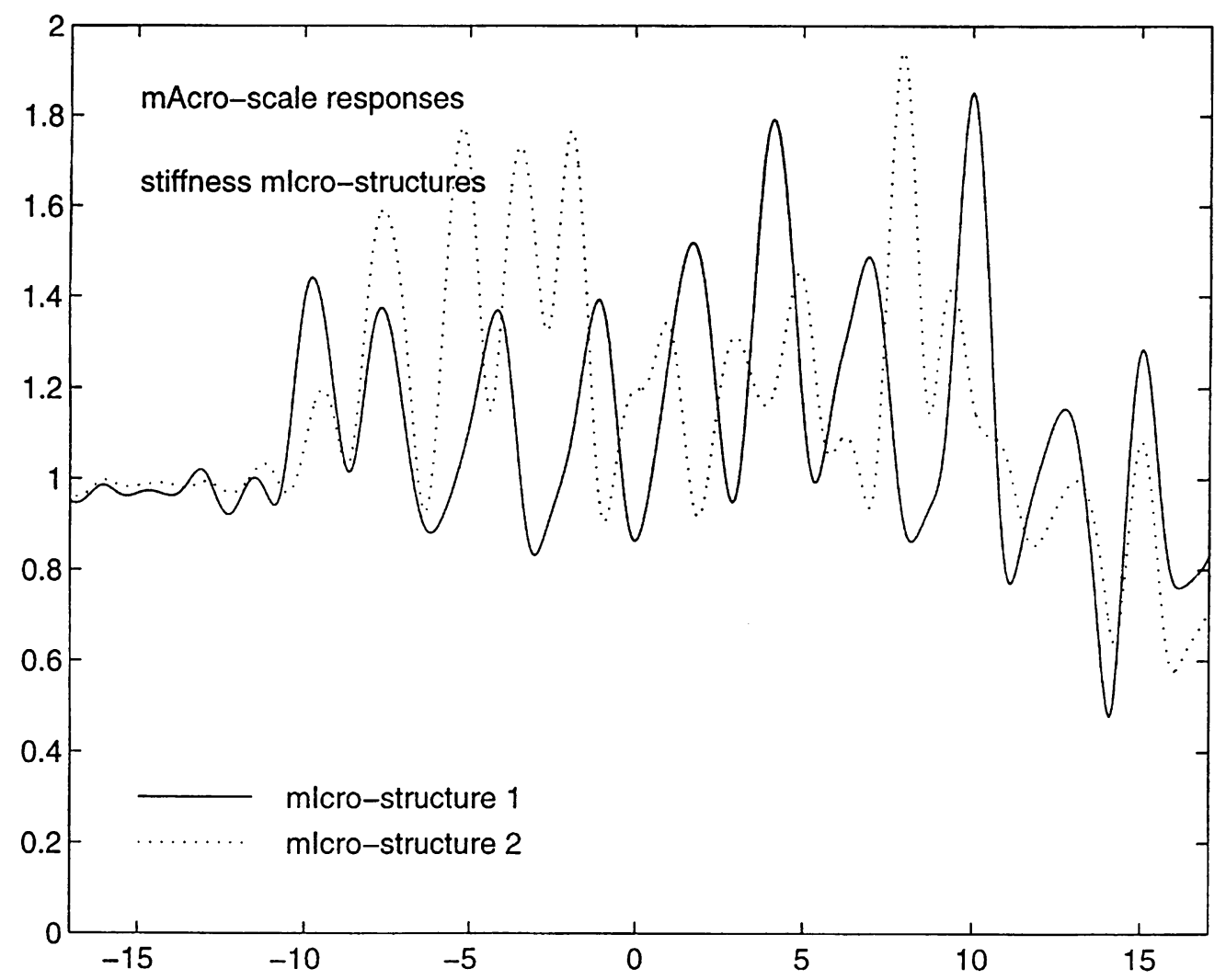

FIG. 11. Magnitude of the macroscale component of the plate response, $u^{s}$, associated with $q_{1}(x)$ (solid line) and with $q_{2}(x)$ (dot line)

which follows from (4.7), and

$$
\left\|T^{d}\right\|_{2} \ll\left\|T^{s}\right\|_{2}
$$

which follows from (4.10b). An effective constitutive relation-a relation written for $u^{s}$ - can now be obtained by reversing (6.2) and using (6.4b),

$$
u(x) \simeq T^{s}(x) / Q(x)=T^{s}(x)\left[\left(\frac{1}{Q(x)}\right)^{s}+\left(\frac{1}{Q(x)}\right)^{d}\right]
$$

where $\left(\frac{1}{Q(x)}\right)^{s, d}$ denote the smooth $(s)$ and detail $(d)$ components of $\frac{1}{Q(x)}$. By taking the smooth part of both sides, we obtain now (a smooth component multiplied by a detail component gives a function with essentially zero local average, i.e., a "detail function"),

$$
u^{s}(x) \simeq T^{s}(x)\left(\frac{1}{Q(x)}\right)^{s} .
$$

This is a local (i.e., algebraic) effective constitutive relation connecting $u^{s}$ and $T^{s}$.

For the task of calculating the plate macroscale response $u^{s}(x)$, the effective constitutive relation should be combined with the integral equation formulation governing $T^{s}(x)$, 


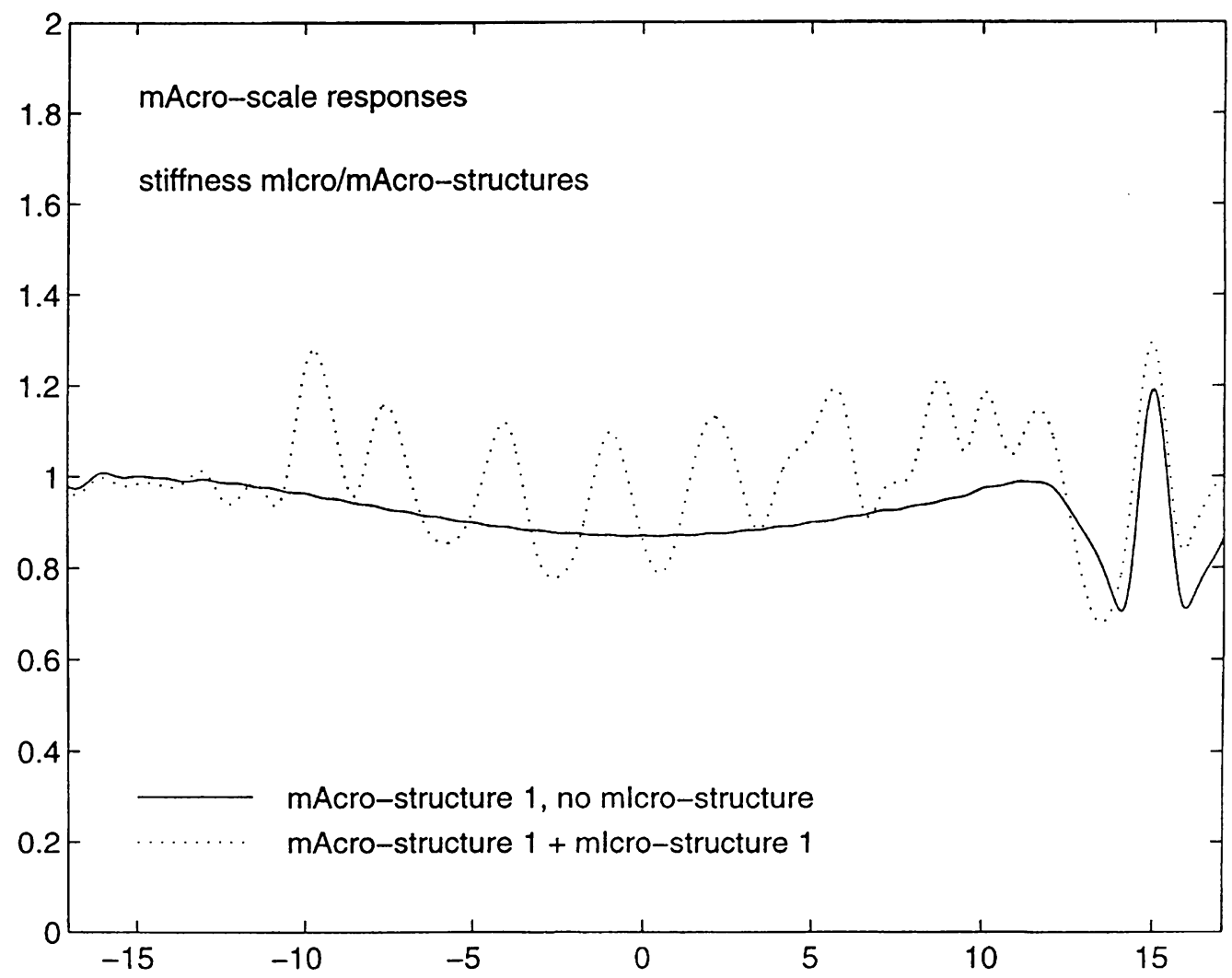

FIG. 12. Magnitude of the macroscale component of the plate response, $u^{s}$, associated with $q_{3}(x)$ (solid line) and with $q_{4}(x)$ (dot line)

given in (6.4a). A noteworthy feature of the latter is that only a smooth measure of the system heterogeneity, namely $r^{s}(x)$, is required.

6.1. An important implication. Microscale stiffness variation can have a significant effect on the macroscale response. This general conjecture-not evidently plausible at first glance-is shown to be correct in Sec. 5.3. Three fundamental questions immediately arise:

1. Does there exist a class of different stiffness microstructures such that the macroscale response associated with each of the members of the class is the same?

2. If the answer is "yes", what is the precise definition of such a class?

3. Again-if the answer is "yes"-can one synthesize an effective stiffness, a smooth stiffness heterogeneity for which the associated complete response is the same as the macroscale response associated with each of the members of the class?

The effective constitutive relation, (6.6), combined with the integral equation, (6.4a), provide the answers; "yes" for 1, then, an algorithmic characterization of the "classes of invariance", and an effective stifiness of the class. The latter is an inherently nonlinear 
process. To derive these results, note that

$$
\frac{1}{Q(x)}=1-r(x)
$$

where $r(x)$ is defined in (6.3c). Then, (6.6) takes on the form

$$
u^{s}(x)=T^{s}(x)[1-r(x)]^{s}=T^{s}(x)\left[1-r^{s}(x)\right] .
$$

Notably, the stiffness heterogeneity measure that affects $T^{s}(x)$ is $r^{s}(x)$ (see (6.4a)). It therefore follows from (6.8) that this is the only stiffneris heterogeneity measure affecting $u^{s}(x)$. Thus, two different microscale variations of $q(x)$, that give the same smoothed $r(x)$, have the same footprint on the macroscale response. A simple synthesis procedure for generating stiffness variations of the same class, as articulated above, follows. Let $r_{1}(x)$ and $r_{2}(x)$ be two functions differing only by their detail components,

$$
r_{i}(x)=r^{s}(x)+r_{i}^{d}(x), \quad i=1,2,
$$

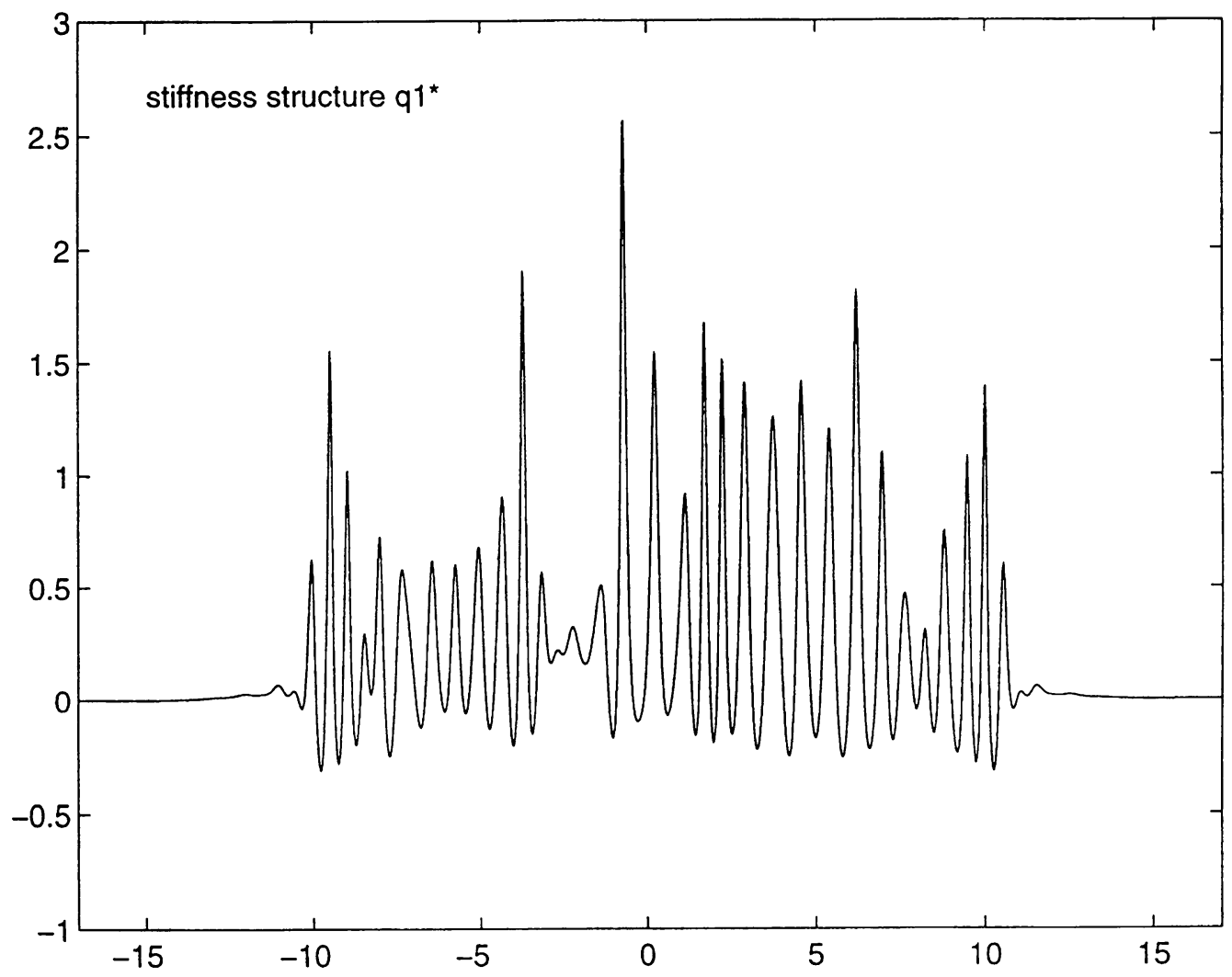

FIG. 13(a). Two different complex structures of stiffness, designed via the procedure of Sec. 6.1 to have the same macroscale response. (a) $q_{1}^{*}(x)$ 
that is,

$$
\begin{aligned}
r_{1}(x) & \neq r_{2}(x), \\
\left(r_{1}(x)\right)^{s} & =\left(r_{2}(x)\right)^{s}=r^{s}(x) .
\end{aligned}
$$

These variations can be obtained by choosing at random a function for $r_{1}(x)$, and adding to it an arbitrary microstructure for $r_{2}(x)$, or, choosing $q_{1}(x)$ for the stiffness variation, computing the corresponding $r_{1}(x)$ via $(6.3 \mathrm{c})$, and performing the articulated step. The associated stiffness variations are obtained by inverting $(6.3 \mathrm{c})$,

$$
q_{i}(x)=r_{i}(x) /\left[1-r_{i}(x)\right], \quad i=1,2 .
$$

Clearly, we have $q_{1}(x) \neq q_{2}(x)$. However, from $(6.9 \mathrm{~b})$ it follows that the associated macroscale responses would be the same. This procedure can be used also to obtain an effective stiffness heterogeneity. For the class defined by (6.9) with $i=1,2,3, \ldots$ (infinitely many different microstructures), the effective stiffness would be that associated with $r^{s}(x)$, i.e., $q_{\mathrm{eff}}(x)=r^{s}(x) /\left[1-r^{s}(x)\right]$. Or, alternatively, the effective stiffness associated

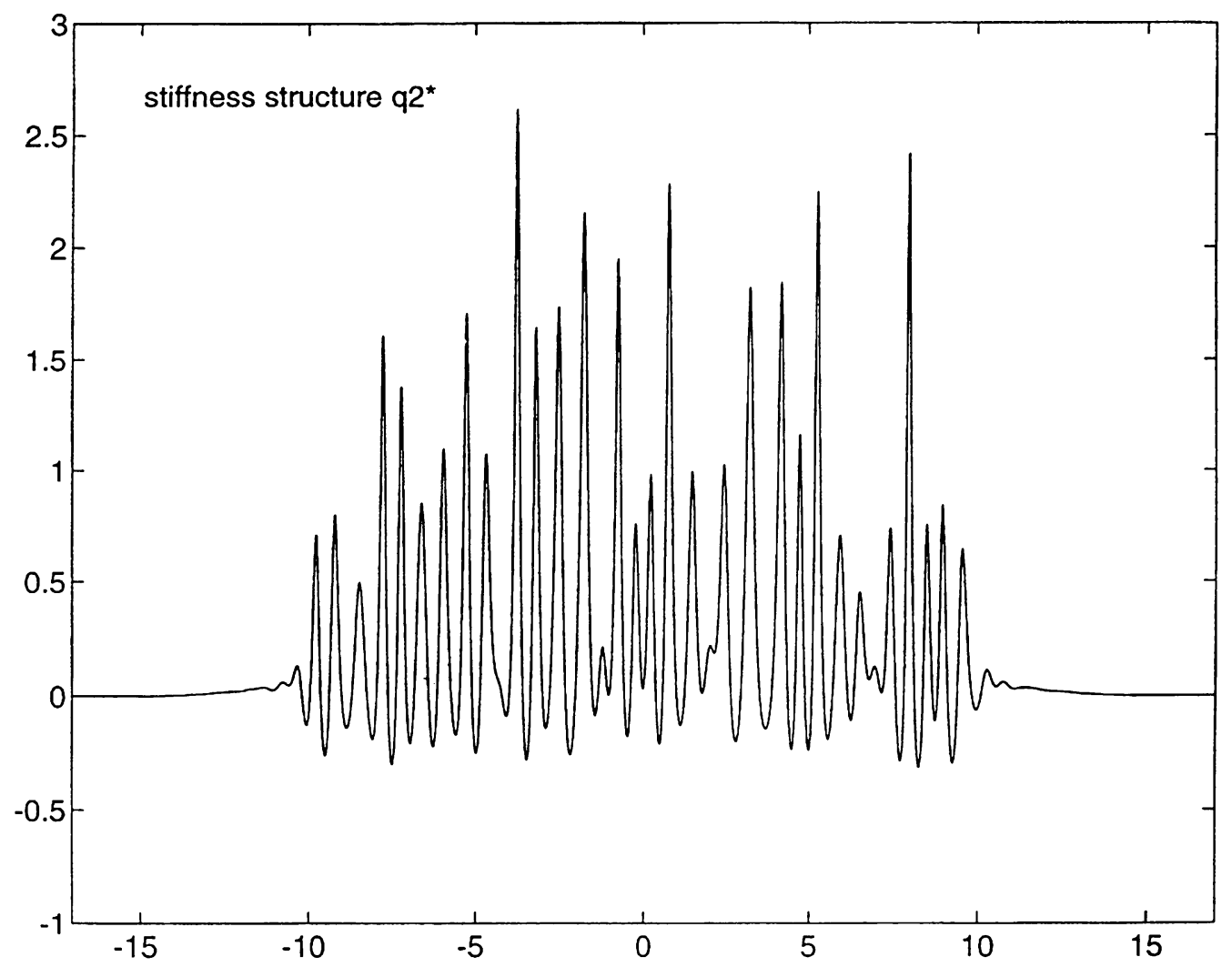

FIG. 13(b). Two different complex structures of stiffness, designed via the procedure of Sec. 6.1 to have the same macroscale response. (b) $q_{2}^{*}(x)$ 


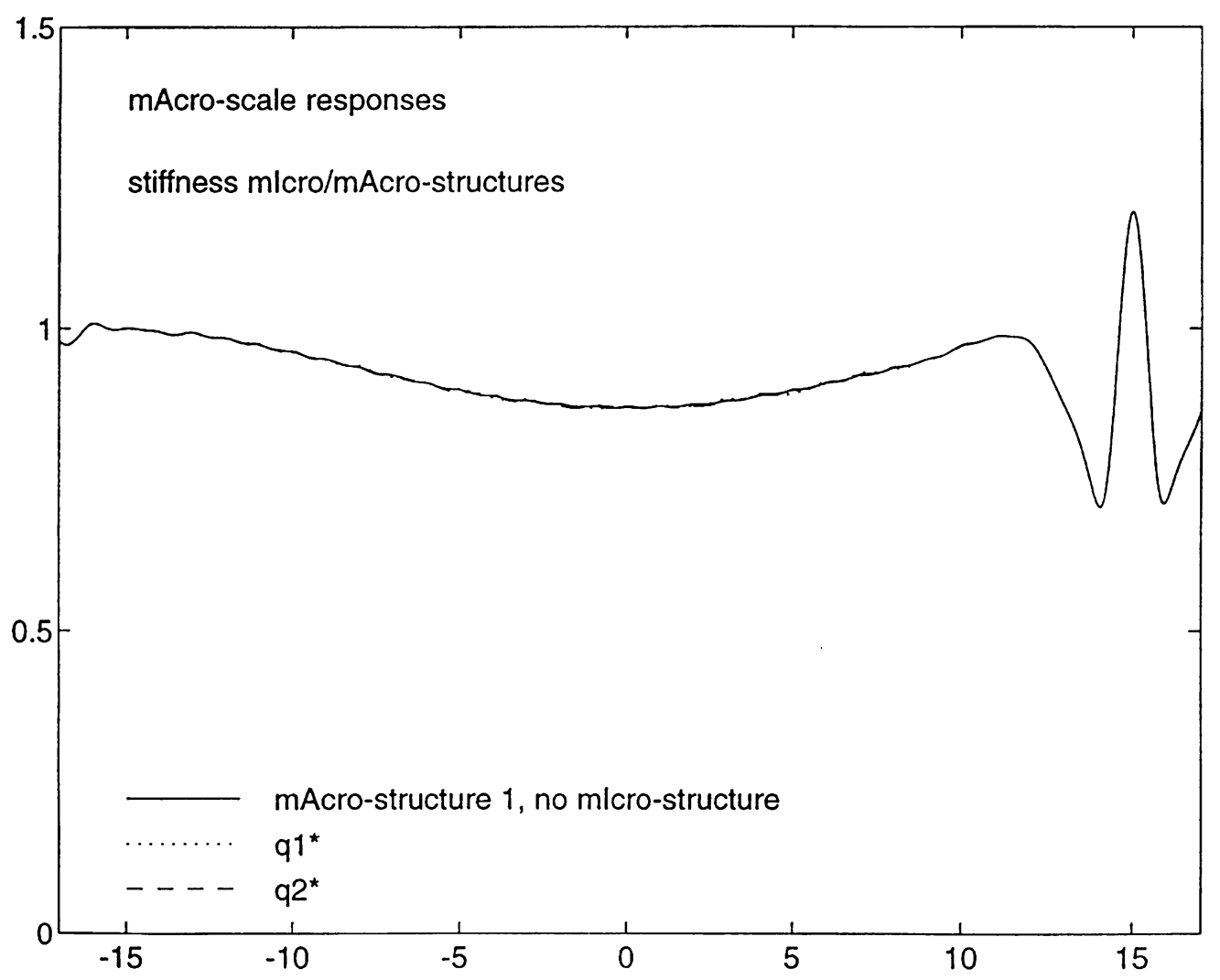

FIG. 14. The macroscale response $u^{s}$ associated with $q_{1}^{*}, q_{2}^{*}$, and $q_{3}=$ $h^{s}$. The three solutions cannot be distinguished to the resolution of this graph.

with the stiffness variation $q_{1}(x)$ (that possesses both smooth and detail components) is obtained by first computing the corresponding $r_{1}(x)$ via (6.3c), then smoothing it via, say, the projection operator of $(2.1 \mathrm{a}) ; r_{1}^{s}(x)=\mathbf{P}_{j} r_{1}(x)$, and finally obtaining $q_{\mathrm{eff}}(x)$ via the inverse of (6.3), $q_{\mathrm{eff}}(x)=r_{1}^{s}(x) /\left[1-r_{1}^{s}(x)\right]$.

6.2. Example. We turn to demonstrate the last results numerically. The procedure described by Eqs. (6.9)-(6.10) and the discussion thereafter is used to synthesize examples of stiffness complex scatterers with identical macroscale response, and with a prescribed effective stiffness. Specifically, we used the macroscale structure $h^{s}(x)$ in Fig. 5(c) as a smooth stiffness, computed the corresponding $r(x)$, added the complex structures $0.6 h_{1}^{d}(x)$ or $0.6 h_{2}^{d}(x)$, and re-inverted the results to get two different complex structures of stiffness $q_{1}^{*}(x)$ and $q_{2}^{*}(x)$, respectively. These are shown in Figs. 13(a) and 13(b). Clearly these stiffness distributions are not the same; they differ in both their macro- and micro-scale components. However, they should have the same macroscale response, and further, it should be identical to that associated with $q_{3}(x)=h^{s}(x)$ of Sec. 5.3 , the latter being the corresponding effective stiffness. Figure 14 shows the macroscale component of the corresponding response field $u^{s}(x)$, subject to the same loading of 
Fig. 10, and compares the results to $u^{s}$ associated with $q_{33}(x)=h^{s}(x)$. The results are indistinguishable within the graphic resolution.

7. Conclusions. We have demonstrated that a small-scale mass density variation has no footprint in the large-scale response of a linearly elastic plate, whereas a smallscale bending stiffness variation does. We showed that the small-scale stiffness variation has no footprint in the large-scale "bending moment" variation in the plate. This was used to identify a condition for a class of small-scale stiffness variations that all lead to the same large-scale plate response.

The simplicity of the two results suggests that there must be easily-grasped underlying physical reasons. These are: first, although the experiment posed is dynamical, this designation applies for large-scale distances. The travel times for distances identified with the small-scale heterogeneities are small when compared to the inverse of the frequency of the time-harmonic forcing. Thus, the interaction of the plate response with the smallscale heterogeneity is essentially statical. This leads to the conclusions that a smallscale mass density variation should have no effect and that the effects of a small-scale stiffness variation should be the same as that calculated for a statical forcing. The second underlying physical reason is that the line-load forcing results in a prediction problem that is one-dimensional; i.e., the plate responds as a beam; and one-dimensional beam theory is statically determinate. That is, the internal stress resultant-the internal bending moment - can be determined from the laws of equilibrium alone. One conclusion of this is that the small-scale material properties of the beam will have no effect in determining the internal bending moment. Thus, the achieved conclusions are consistent with an intuitive understanding of the underlying physics; this consistency is certainly satisfying.

This consistency also allows speculation as to the applicability of the results for a broader class of problems. One speculation is the conclusion that the small-scale mass density has no footprint in the large-scale response, has a broader applicability. The argument that the reason lies in the observation that the interaction of the structure response and the structure heterogeneity is statical, is very robust. The observation that the absence of a footprint of the small-scale stiffness heterogeneity in a large-scale measure of internal stress lies in the fact that the statical problem is determinate, using the laws of equilibrium, and suggests this conclusion to be idiosyncratic to the specific experiment. In two dimensions, for example, the state of the internal stress in a plate is statically indeterminate. Thus, this internal distribution of stress will depend on the material heterogeneity. This does not necessarily mean that there does not exist a class of stiffness heterogeneity, all of which will lead to the same large-scale structure response. It does suggest, however, that the conditions for describing this class will be more complex than the simple formula achieved.

Both of these speculations are consistent with our understanding of the micromechanics of composite materials. A more convincing demonstration awaits the application of the multiresolution decomposition and the phase-space substructuring methodology to a more complicated system. 
Appendix: Bound estimates for the matrix norms. The results given in (4.6a)(4.6c) are derived here. The discussion is limited to the scatterer defined by $(4.2)-(4.3 \mathrm{~b})$, with the scales hierarchy articulated by (4.1). Thus, (4.4a)-(4.5b) apply. Further, with the smoothness of $G$,

$$
\left|G_{M}(x)\right| \leq \lambda^{-M}
$$

It is essential to recognize the scatterer outer dimension as measured on the scale of $\lambda$. Thus, denoting by $N_{0}$ the total number of resolution zero grid points spanning the scatterer extent, we have (see (4.1))

$$
N_{0}=2^{-m_{a}} O(1)
$$

A.1. Bound estimate for $\|\Psi\|$. With $(4.4 \mathrm{~b}),(4.5 \mathrm{~b})$, and the scatterer characterization (4.2)-(4.2b), one has

$$
\Psi_{m^{\prime} n^{\prime}, m n} \simeq \beta b_{n} 2^{-m^{\prime}(M+1 / 2)} G_{M}\left(x_{m^{\prime} n^{\prime}}-x_{m n}+2^{-m^{\prime}-1}-2^{-m-1}\right) \delta_{m, m_{i}-1}
$$

where $\delta_{i j}$ is the Kronecker delta function. Using (A.1)

$$
\left|\Psi_{m^{\prime} n^{\prime}, m n}\right| \leq \beta\left|b_{n}\right| 2^{-m^{\prime}(M+1 / 2)} \lambda^{-M} \delta_{m, m_{i}-1} .
$$

With the Euclidean matrix norm inequality $\|\Psi\|^{2} \leq \sum_{m^{\prime}, n^{\prime}, m, n}\left|\Psi_{m^{\prime} n^{\prime}, m n}\right|^{2}$, one has

$$
\begin{aligned}
\|\boldsymbol{\Psi}\|^{2} & \leq \beta^{2} \lambda^{-2 M} \sum_{m^{\prime}, n^{\prime}, n}\left|b_{n}\right|^{2} 2^{-2 m^{\prime}(M+1 / 2)} \quad \text { using (A.4) } \\
& =\beta^{2} \lambda^{-2 M} \sum_{m^{\prime}, n^{\prime}} 2^{-2 m^{\prime}(M+1 / 2)} \quad u \operatorname{sing}(4.3 \mathrm{~b})
\end{aligned}
$$

The summation over $n^{\prime}$ depends on $m^{\prime}$, since the number of grid points associated with the resolution $m^{\prime}$ scales like $N_{0} 2^{m^{\prime}}$, where $N_{0}$ is the number of points at resolution 0 . Thus, with (A.2),

$$
\begin{aligned}
\|\boldsymbol{\Psi}\|^{2} & \leq \beta^{2} \lambda^{-2 M} 2^{-m_{a}} \sum_{m^{\prime}=j}^{\infty} 2^{-2 M m^{\prime}} \\
& =\beta^{2} \lambda^{-2 M} 2^{-m_{a}} 2^{-2 M_{j}}\left(1-2^{-2 M}\right)^{-1} .
\end{aligned}
$$

The last result can be rewritten as

$$
\|\boldsymbol{\Psi}\|^{2} \leq \beta^{2} \lambda\left(1-2^{-2 M}\right)^{-1}\left(\lambda 2^{m_{a}}\right)^{-1}\left(\lambda 2^{j}\right)^{-2 M} .
$$

However, by multiplying (4.1) with $2^{j}$ one finds $\lambda 2^{j} \gg 1$ and by multiplying it with $2^{m_{a}}$ one finds $\lambda 2^{m_{a}} \approx 1$. In addition, recall $|\beta| \ll 1$ and usually $M \geq 2$. Thus, for finite $\lambda$ we obtain the result in (4.6a).

A.2. Bound estimate for $\|\overline{\mathbf{C}}\|$. With $(4.4 \mathrm{~d}),(4.5 \mathrm{~b})$, and the scatterer characterization $(4.2)-(4.2 \mathrm{~b})$,

$$
\bar{C}_{m^{\prime} n^{\prime} . n} \simeq \beta a_{n} 2^{-m^{\prime}(M+1 / 2)} G_{M}\left(x_{m^{\prime} n^{\prime}}+2^{-m^{\prime}-1}-x_{j n}\right) .
$$


Thus, with the norm inequality $\|\overline{\mathbf{C}}\|^{2} \leq \sum_{m^{\prime} . n^{\prime} . n}\left|\bar{C}_{m^{\prime} n^{\prime} . m n}\right|^{2}$, one has

$$
\begin{aligned}
\|\overline{\mathbf{C}}\|^{2} & \leq \beta^{2} \lambda^{-2 M} \sum_{m^{\prime} \cdot n^{\prime} \cdot n}\left|a_{n}\right|^{2} 2^{-2 m^{\prime}(M+1 / 2)} \quad u \operatorname{sing}(\text { A.8) and (A.1) } \\
& =\beta^{2} \lambda^{-2 M} \sum_{m^{\prime} \cdot n^{\prime}} 2^{-2 m^{\prime}(M+1 / 2)} \quad u \operatorname{sing}(4.3 \mathrm{~b}) .
\end{aligned}
$$

The inequality (4.6b) follows by repeating the steps of (A.6a)-(A.7).

A.3. Bound estimate for $\|\mathbf{C}\|$. With (4.4c), (4.5a), and the scatterer characterization,

$$
C_{n^{\prime} \cdot m n} \simeq 2^{-j / 2} b_{n} G\left({ }_{j n^{\prime}}-x_{m n}-2^{-m-1}\right) \delta_{m \cdot m_{i}-1} .
$$

Thus, by (A.10) and (A.1),

$$
\|\mathbf{C}\|^{2} \leq \sum_{n^{\prime} \cdot m \cdot n}\left|c_{n^{\prime} \cdot m n}\right|^{2} \leq 2^{-j} \sum_{n \cdot n^{\prime}}\left|b_{n}\right|^{2}
$$

The summation over $n$ is $O(1)$ by the scatterer characterization (4.3b). The $n^{\prime}$ index counts the grid points at resolution $j$ (see matrix construction in Fig. 3). The latter is $O\left(2^{j}\right)$, and hence the result in (4.6c).

\section{REFERENCES}

[1] B. Z. Steinberg and J. J. McCoy, Towards local effective parameter theories using multiresolution decomposition, J. Acoust. Soc. Amer. 96, 11301143 (1994)

[2] B. Z. Steinberg and J. J. McCoy, Effective measures for nonstationary microscale stiffness variation using multiresolution decomposition, J. Acoust. Soc. Amer. 98, 3516-3526 (1995)

[3] S. G. Mallat, A theory for multiresolution signal decomposition: The wavelet representation, IEEE Trans. Patt. Anal. Mach. Intel. 11 (7), 674693 (1989)

[4] S. G. Mallat, Multiresolution approximations and wavelet orthonormal bases of $L_{2}$, Trans. Amer. Math. Soc. 315, $69-87$ (1989)

[5] I. Daubechies, Ten lectures on wavelets, CBMS-NSF Series in Applied Mathematics, SIAM Publ., Philadelphia, 1992

[6] M. E. Brewster and G. Beylkin, A multiresolution strategy for numerical homogenization, Appl. Comp. Harmonic Anal. 2, 327349 (1995)

[7] B. Z. Steinberg, A multiresolution theory of scattering and diffraction, Wave Motion 19, 213-232 (1994)

[8] G. Beylkin, R. Coifman, and V. Rokhlin, Fast wavelet transforms and numerical algorithms I, Commun. Pure Appl. Math. 44, 141-183 (1991)

[9] B. Z. Steinberg and Y. Leviatan, On the use of the wavelet expansion in the method of moments, IEEE Trans. Antennas and Propagation, May 1993 